\title{
Understanding Portfolio Efficiency with Conditioning Information*
}

\author{
Francisco Peñaranda \\ Universitat Pompeu Fabra and Barcelona GSE \\ $<$ francisco.penaranda@upf.edu>
}

Revised: October 2011

\begin{abstract}
We show that unconditionally efficient returns do not achieve the maximum unconditional Sharpe ratio, neither display zero unconditional Jensen's alphas, when returns are predictable. Next, we define a new type of efficient returns that is characterized by those unconditional properties. We also study a different type of efficient returns that is rationalized by standard mean-variance preferences and motivates new Sharpe ratios and Jensen's alphas. We revisit the testable implications of asset pricing models from the perspective of the three sets of efficient returns. We also revisit the empirical evidence on the conditional variants of the CAPM and the Fama-French model from a portfolio perspective.
\end{abstract}

Keywords: Conditional CAPM, Dynamic portfolio strategies, Jensen's alpha, Mean-variance frontiers, Representing portfolios, Sharpe ratio.

JEL: C12, G11, G12

\footnotetext{
${ }^{*}$ I thank seminar participants at Universidad Carlos III, CEMFI, Foro de Finanzas (Barcelona, 2008), IE Business School, MFA (Las Vegas, 2010) and LSE, and specially Urooj Amjad, Geert Bekaert, Amit Goyal, Stefan Nagel, José Penalva, Julián Pérez-Alzueta, Cesare Robotti, Enrique Sentana and Jay Shanken, for helpful comments and suggestions. Financial support from the Spanish Ministry of Education and Science through grant ECO2008-03066 and the Government of Catalonia is gratefully acknowledged.
} 


\section{Introduction}

The seminal paper of Hansen and Richard (1987) analyzed the tension between the conditional implications of asset pricing theory and the use of unconditional moments in empirical work, as these moments are easily estimated with sample averages. They developed a theoretical framework to study the mean-variance implications of asset pricing models and the effect of information omission. They proved that unconditionally efficient (UE) returns are a subset of the conditionally efficient (CE) returns. Hence the conditional CAPM implies that the market portfolio is CE, but not necessarily UE.

This paper extends their results. Our main contribution is a comprehensive analysis of three different types of $\mathrm{CE}$ returns. $\mathrm{CE}$ returns have conditional properties similar to the unconditional properties of the textbook Markowitz (1952) frontier. The mean-variance frontier is linear with a safe asset, providing a unique risk-return trade-off defined by the Sharpe ratio on the frontier. Moreover, if we use a return on the frontier as a factor to price any feasible return then the corresponding Jensen's alpha is zero.

In contrast, we show that UE returns ${ }^{1}$ do not satisfy any of those textbook properties in an unconditional sense unless the safe asset return is constant. In general the safe return (e.g., the Treasury-bill rate) is risky from the perspective of unconditional moments, which distorts the properties of UE returns in terms of unconditional Sharpe ratios and Jensen's alphas. To the best of our knowledge, these properties of UE returns have not been studied before, ${ }^{2}$ even though unconditional measures are commonly used in empirical finance. Lewellen and Nagel (2006) analyze the unconditional alphas of CE returns, which are not zero in general even though their conditional alphas must be zero. They do not study UE returns in particular.

These facts open the question of what type of efficiency is actually tested in empirical work. We characterize a new set of efficient returns that achieve the maximum unconditional Sharpe ratio or equivalently display zero unconditional Jensen's alphas. For this reason, we refer to this new subset of CE returns as performance efficient (PE) returns. We show that PE returns minimize the tracking error with respect to the safe asset return instead of the total return variance. Ferson and Siegel (2009) construct an efficiency test ${ }^{3}$ that is based on the maximum

\footnotetext{
${ }^{1}$ Several papers have used UE returns to guide portfolio choice. See e.g. Ferson and Siegel (2001) and, adding a benchmark, Chiang (2009). Other papers such as Brandt and Santa-Clara (2006) and Bansal, Dahlquist, and Harvey (2004) approximate UE returns through managed portfolios.

${ }^{2}$ Hansen and Richard (1987) study a general set-up that may or not include a safe asset, but they only make explicit the role of a safe asset to clarify some ideas, such as the safe return is CE but not necessarily UE.

${ }^{3}$ Other examples of recent references are Wang (2003) and Lewellen and Nagel (2006). See also the references
} 
unconditional Sharpe ratio with conditioning information. Our results point out that these authors are testing if a particular return is PE instead of UE. They assume a constant safe asset return when they develop their test, and in that case both types of efficiency are equivalent. However, the safe asset return has a non-negligible variance for the purposes of testing efficiency and asset pricing models as our empirical application shows.

We also study a third type of efficient returns, which represents a different subset of $\mathrm{CE}$ returns, and can be rationalized by mean-variance preferences commonly used in finance. If we decompose the unconditional variance as the average conditional variance plus the variance of the conditional mean then these optimal returns minimize the first component only. We use the term residually efficient (RE) returns because the average conditional variance is equal to the variance of a residual, the return minus its conditional mean. Unlike UE returns, RE returns inherit the properties of the Markowitz frontier in terms of alphas and Sharpe ratios, if the required variances and covariances are based on the residuals instead of the returns themselves.

Our theoretical contributions conclude with important results on testing mean-variance efficiency and asset pricing models. ${ }^{4}$ Empirical finance is often interested in testing that some portfolio of a given set of returns lies on the efficient part of the mean-variance frontier spanned by a wider set of returns. We show that, for any of the commented efficiencies we may be interested in (CE, UE, PE, or RE), the null hypothesis is the same and it is equivalent to zero conditional alphas. However, if we think of the CAPM for instance, and we also want to impose that the market return itself is $\mathrm{PE}$ or $\mathrm{RE}$ (which is equivalent to a stochastic discount factor with a constant weight on the market) then the null hypothesis adds predictability constraints of different types on the market return.

To illustrate our theoretical results and show their empirical relevance, we study conditional variants of the CAPM and the Fama-French model. We use four excess returns: the excess return on the market, two excess returns that capture the size and value effects, and another one that captures the momentum effect. We use three prominent predictors, the dividend price ratio, the default spread, and the term spread. We find return predictability in these data, with considerable differences across the three types of efficiency and time-variation in the weights of efficient returns. This time-variation invalidates asset pricing models with fixed-weight stochastic

therein as a comprehensive review of papers taking into account conditioning information in empirical work, testing the conditional CAPM, etc.

${ }^{4}$ Sentana (2009) surveys portfolio efficiency tests without conditioning information. 
discount factors. The value and momentum effects are far more relevant than the size effect, and also their weights in optimal portfolios tend to be higher than the market return weights. Hence we find that the value effect is more important than the size effect as a source of rejection of the CAPM, and the Fama-French model cannot price momentum. These patterns are in line with the empirical literature, see Schwert (2003) for instance.

In our empirical application the unconditional Sharpe ratio of UE returns changes considerably for target returns around the safe asset. As we increase the target, this Sharpe ratio converges towards its value for PE returns, which is its maximum value. The unconditional Sharpe ratio of PE returns is considerably higher than the ratio for fixed-weight returns, which proves the benefits from using conditioning information. Nevertheless, there are regions in meanvariance space where fixed-weight portfolios perform better than some types of efficient returns. In addition, the correct zero-beta return of UE returns can be very different from the average safe asset return. However, this is not the usual approach in empirical work, where the safe asset is directly used in the computations of unconditional alphas instead. For UE returns, these alphas can be very different from zero, the value that characterizes PE returns instead, and also depend on the particular target return.

The rest of the paper is organized as follows. Section 2 describes the theoretical framework and the empirical application that illustrates our theoretical results. We provide a deep analysis of three types of efficient returns in Section 3. Next, we develop the implications for testing asset pricing models in Section 4. Finally, we present our conclusions in Section 5. Proofs and auxiliary results are gathered in the appendix.

\section{Theoretical and Empirical Set-up}

In our investment set-up there are $N+1$ unit-cost payoffs, the safe asset return $R_{0}$ and $N$ risky assets whose random returns $\mathbf{R}=\left(R_{1}, \ldots, R_{N}\right)^{\prime}$ are defined on an underlying probability space. We define the corresponding vector of excess returns as $\mathbf{A}=\left(R_{1}-R_{0}, \ldots, R_{N}-R_{0}\right)^{\prime}$. We follow the theoretical framework in Hansen and Richard (1987) to analyze portfolio strategies when returns are predictable. Let $G$ denote the investors' information when trading, typically containing signals that are informative about future asset payoffs. We denote the set of all random variables that are measurable with respect to $G$ by $I$. The safe asset return $R_{0}$ and the 
first two conditional moments of the vector of excess returns, which are denoted by

$$
E(\mathbf{A} \mid G), \quad E\left(\mathbf{A A}^{\prime} \mid G\right)
$$

respectively, belong to $I$. To ease the exposition, we assume the smallest eigenvalue of $\operatorname{Var}(\mathbf{A} \mid G)=$ $E\left(\mathbf{A A}^{\prime} \mid G\right)-E(\mathbf{A} \mid G) E(\mathbf{A} \mid G)^{\prime}$ is uniformly bounded away from 0 with probability one. This implies that none of the primitive risky assets is actually conditionally riskless or redundant. We also assume $E(\mathbf{A} \mid G) \neq \mathbf{0}$ to avoid trivial mean-variance frontiers.

Investors can condition their portfolio weights on the information given by $G$, i.e. they can construct portfolio strategies with weights that belong to $I$. We will be interested in two subsets of the corresponding payoffs, the unit-cost strategies (or gross returns) and the zero-cost strategies (or arbitrage portfolios), which we denote by $\mathcal{R}$ and $\mathcal{A}$ respectively. We refer to their elements as $R \in \mathcal{R}$ and $A \in \mathcal{A}$. If an investor is endowed with some positive wealth, which we can normalize to 1 without loss of generality, then she will only be interested in portfolio strategies that cost 1 for every possible value of the signals in $G$.

There are two arbitrage portfolios that play a key role in the characterization of the frontiers. $A^{+}$represents the conditional mean of arbitrage portfolios with an uncentred second moment, while $A^{++}$uses a centred second moment: $A^{+}$is the unique arbitrage portfolio that satisfies

$$
E\left(A^{+} A \mid G\right)=E(A \mid G)
$$

and $A^{++}$is the unique arbitrage portfolio that satisfies

$$
\operatorname{Cov}\left(A^{++}, A \mid G\right)=E(A \mid G)
$$

for every $A \in \mathcal{A}$. These representing portfolios are conditionally proportional,

$$
A^{++}=\left[1-E\left(A^{+} \mid G\right)\right]^{-1} A^{+},
$$

and can be expressed explicitly as

$$
A^{+}=\mathbf{A}^{\prime}\left[E\left(\mathbf{A A}^{\prime} \mid G\right)\right]^{-1} E(\mathbf{A} \mid G), \quad A^{++}=\mathbf{A}^{\prime}[\operatorname{Var}(\mathbf{A} \mid G)]^{-1} E(\mathbf{A} \mid G)
$$

in our investment set-up. ${ }^{5}$

\footnotetext{
${ }^{5}$ Their conditional and unconditional means are $E\left(A^{+} \mid G\right)=E(\mathbf{A} \mid G)^{\prime}\left[E\left(\mathbf{A A}^{\prime} \mid G\right)\right]^{-1} E(\mathbf{A} \mid G), \quad E\left(A^{++} \mid G\right)=E(\mathbf{A} \mid G)^{\prime}[\operatorname{Var}(\mathbf{A} \mid G)]^{-1} E(\mathbf{A} \mid G)$, and$$
E\left(A^{+}\right)=E\left[E(\mathbf{A} \mid G)^{\prime}\left[E\left(\mathbf{A} \mathbf{A}^{\prime} \mid G\right)\right]^{-1} E(\mathbf{A} \mid G)\right], \quad E\left(A^{++}\right)=E\left[E(\mathbf{A} \mid G)^{\prime}[\operatorname{Var}(\mathbf{A} \mid G)]^{-1} E(\mathbf{A} \mid G)\right],
$$
respectively.
} 
To illustrate our theoretical results and show their empirical relevance, we use return data from Ken French's Data Library. In particular, we use the Treasury-Bill return as $R_{0}$, and up to four excess returns as $\mathbf{A}$. The first entry is the excess return on the market (MMR). The next two entries are the Small-Minus-Big (SMB) and High-Minus-Low (HML) excess returns, which are constructed by means of six size and book-to-market sorted portfolios. SMB goes long in small capitalization stocks and short in big ones, while HML goes long in high book-to-market stocks and short in low ones. Finally, the Winners-Minus-Losers (WML) excess return, goes long in recent winner, or high return stocks, and short in loser ones. ${ }^{6}$ See Ken French's web page, as well as Fama and French (1993), for further details. We compare three sets of available assets, the first one is given by the safe asset and MMR, the second one adds SMB and HML, and the third one also includes WML. We use annual data from 1954 to 2010 in the main text, and leave monthly data to Appendix B.

We use three prominent predictors, the US dividend price ratio (DP), the default spread (DS), and the term spread (TS). DP is taken from Robert Shiller's web page, while DS and TS are constructed from FRED data; in particular from yields on AAA and BAA-rated bonds, and from 10 and 1-year constant maturity Treasury bond yields respectively. These predictors are widely used in empirical finance, see Ferson and Siegel (2009) for instance.

We keep the number of returns and predictors low to show that our theoretical results do not require many returns and/or predictors to be quantatively important. Similarly, our model of conditional moments is simple to guarantee that our evidence does not depend on a complex set-up. In the case of annual data, $E(\mathbf{A} \mid G)$ is linear in the predictors, following the spirit of predictive regressions, and $\operatorname{Var}(\mathbf{A} \mid G)$ is constant as GARCH effects are weak at this frequency. Nevertheless, we allow time variation in $\operatorname{Var}(\mathbf{A} \mid G)$ with monthly data in the appendix. Table 1 describes our empirical set-up.

$<$ Table 1: Description of annual data $>$

In panel $\mathrm{B}$, the $R^{2}$ of MMR is clearly high when compared to SMB or HML, but WML is the excess return with the highest $R^{2}$. On the other hand, the Wald test of zero slopes for MMR represents the strongest rejection of lack of predictability, followed by SMB and WML. We also

\footnotetext{
${ }^{6}$ The momentum premium, introduced by Jegadeesh and Titman (1993), has become one of the strongest anomalies from the perspective of both the CAPM and the Fama-French model without conditioning information.
} 
reject that all the slopes of the four excess returns are jointly zero. Panel $\mathrm{C}$ shows that the weights of $A^{+}$and $A^{++}$have standard deviations of similar order to their means, which is another signal on the relevance of return predictability in this data set. Nevertheless, the average of the weights of $A^{+}$is not very different from the weights of its fixed-weight counterpart, a portfolio that uses unconditional moments in (4) instead. Figure 1 displays the time series of these weights.

$<$ Figure 1: Weights of representing portfolios $>$

There is more variability in the weights of $A^{++}$. In general $E\left(A^{+} \mid G\right)$ must be bounded by 1, while $E\left(A^{++} \mid G\right)$ is not bounded. Furthermore, in our simple model, the weights of $A^{++}$ are linear in the predictors, while the weights of $A^{+}$are given by the product of the inverse of matrix that is quadratic in the predictors times the linear risk premia. The conservativeness of the second type of weights in the face of extreme signals is emphasized by Ferson and Siegel (2001) in terms of robustness. Note that in such a simple set-up these portfolio weights are constant if and only if all the predictive regression slopes are zero. ${ }^{7}$ Table 1 rejected the lack of predictability. Finally, Note that HML and WML tend to take higher values than MMR, while SMB usually has the lowest weights.

\section{Efficient Returns with Conditioning Information}

The conditionally efficient (CE) returns are defined by Hansen and Richard (1987) as the returns with minimum conditional variance $\operatorname{Var}(R \mid G)$ for a given target of conditional expected returns $E(R \mid G)$. That is, the set of returns that solve the optimization problem

$$
\min _{R \in \mathcal{R}} E\left(R^{2} \mid G\right) \text { for a given } E(R \mid G) \in I
$$

which we denote by $R_{C}$. Similarly, we denote by $A_{C}$ the excess returns that solve the problem

$$
\min _{A \in \mathcal{A}} E\left(A^{2} \mid G_{1}\right) \text { for a given } E(A \mid G) \in I .
$$

CE excess returns can be represented $a^{8}$

$$
A_{C}=\frac{E\left(A_{C} \mid G\right)}{E\left(A^{+} \mid G\right)} A^{+}=\frac{E\left(A_{C} \mid G\right)}{E\left(A^{++} \mid G\right)} A^{++},
$$

\footnotetext{
${ }^{7}$ Richer models of conditional moments may allow return predictability and, at the same time, constant weights in one of the representing portfolios.

${ }^{8}$ The proof follows the arguments in Hansen and Richard (1987), using the mean square inner product $E(x y \mid G)$ between random variables $x$ and $y$, and the associated mean square norm $\sqrt{E\left(x^{2} \mid G\right)}$.
} 
and $\mathrm{CE}$ returns can be represented as ${ }^{9}$

$$
R_{C}=R_{0}+A_{C}
$$

for some $A_{C}$. In the very special case of all risk premia being zero then $A^{+}=0$, and the $\mathrm{CE}$ returns collapse to the singleton $R_{0}$. We assumed the more plausible case $A^{+} \neq 0$ in the paper.

The conditional Sharpe ratio of an excess return $A$ different from 0 is defined as

$$
S=E(A \mid G) / \sqrt{\operatorname{Var}(A \mid G)}
$$

and the conditional Jensen's alpha of an excess return $A$ with respect to the pricing factor $A_{\beta}$ (an excess return different from 0 ) is defined as

$$
\alpha=E(A \mid G)-\beta E\left(A_{\beta} \mid G\right), \quad \beta=\operatorname{Cov}\left(A, A_{\beta} \mid G\right) / \operatorname{Var}\left(A_{\beta} \mid G\right) .
$$

We can use the same expressions with returns $R$ after subtracting the safe return.

CE excess returns different from 0 are characterized by achieving the maximum $S^{2}=$ $E\left(A^{++} \mid G\right)$. They are also characterized by $\alpha=0$ when they are used as a factor to price any $A \in \mathcal{A}$. Given the representation (8), CE returns satisfy the same properties as CE excess returns in terms of $S^{2}$ and $\alpha$. These results can be interpreted as a translation of the Markowitz frontier properties to conditional moments. The link between portfolio efficiency and zero alphas has been widely used in the Markowitz set-up. For instance, see Gibbons, Ross and Shanken (1989) and the references therein.

The CE returns lie along two straight lines on the $[\sqrt{\operatorname{Var}(R \mid G)}, E(R \mid G)]$ space for each possible value of the signals in $G$, and those two lines intersect on the vertical axis at $R_{0}$. The addition of a safe asset when moving from $\mathcal{A}$ to $\mathcal{R}$ does not change the optimal risk-return trade-off $\sqrt{E\left(A^{++} \mid G\right)}$, as any $R_{C}$ can be expressed as an $A_{C}$ plus $R_{0}$. Note that $E\left(A^{++} \mid G\right)$ is a quadratic function of the predictors in our simple model of linear risk premia and constant variances. Hence the Sharpe ratio is constant if and only if there is not predictability in this simple model. Lack of predictability was rejected in Table 1.

\footnotetext{
${ }^{9}$ This expression specializes the representation in Hansen and Richard (1987) to the existence of a safe asset. Their representation becomes

which can also be expressed as

$$
R_{C}=R_{0}\left(1-A^{+}\right)+\omega A^{+}, \quad \omega \in I,
$$$$
R_{C}=R_{0}+\left(\omega-R_{0}\right) A^{+}=R_{0}+A_{C}
$$

by choosing the $A_{C}$ associated to $E\left(A_{C} \mid G\right) / E\left(A^{+} \mid G\right)=\omega-R_{0}$. We can also represent the CE returns in the spirit of Chamberlain and Rothschild (1983),

$$
R_{C}=R_{0}+w A^{++}, \quad w \in I,
$$

which can also be expressed as $R_{0}+A_{C}$ by choosing the $A_{C}$ associated to $E\left(A_{C} \mid G\right) / E\left(A^{++} \mid G\right)=w$.
} 
Figure 2 displays the time series of the safe asset return and the conditional Sharpe ratio of $\mathrm{CE}$ returns in our empirical application. The mean of the safe asset return is 4.9307 and its standard deviation is 2.9398. The dynamics of the Sharpe ratio are similar across the three sets of returns, and it is clear that WML increases considerably the level of the ratio. If the only available risky return is MMR then the average of the Sharpe ratio is 0.493 and its standard deviation is 0.3245 . If we add SMB and HML, then the average increases to 0.7884 and the standard deviation to 0.4118. After the inclusion of WML, the average jumps to 1.4045 and the standard deviation increases to 0.4975 .

$<$ Figure 2: Safe asset return and conditional Sharpe ratio of CE returns $>$

\subsection{Unconditionally Efficient (UE) Excess Returns}

Unconditional moments, estimated by sample averages, are often used in empirical work to avoid the potential misspecification of models of conditional moments. In addition, in many practical situations the observer of the agents' decisions only has access to an information set that is much coarser than the agents' information set. The performance evaluation of a portfolio manager is a typical example of the use of unconditional moments by an outside evaluator who may not have access to the proprietary strategies followed by the manager.

These issues motivate the analysis of UE returns and excess returns. We denote by $A_{U}$ the excess returns that solve the problem

$$
\min _{A \in \mathcal{A}} E\left(A^{2}\right) \text { for a given } E(A) \in \mathbb{R} .
$$

The following proposition studies the properties of UE excess returns. The unconditional Sharpe ratio of an excess return $A$ is defined as

$$
S_{U}=E(A) / \sqrt{\operatorname{Var}(A)}
$$

and the unconditional Jensen's alpha of an excess return $A$ with respect to a pricing factor $A_{\beta}$ is defined as

$$
\alpha_{U}=E(A)-\beta_{U} E\left(A_{\beta}\right), \quad \beta_{U}=\operatorname{Cov}\left(A, A_{\beta}\right) / \operatorname{Var}\left(A_{\beta}\right) .
$$

Proposition 1 Representation and properties of UE excess returns defined by (9):

1. UE excess returns can be represented as

$$
A_{U}=\frac{E\left(A_{U}\right)}{E\left(A^{+}\right)} A^{+}
$$


2. UE excess returns different from 0 are characterized by achieving the maximum

$$
S_{U}^{2}=\frac{E\left(A^{+}\right)}{1-E\left(A^{+}\right)} .
$$

They are also characterized by

$$
\alpha_{U}=0
$$

when they are used as a factor to price any $A \in \mathcal{A}$.

There is a single optimal risk-return trade-off on the $[\sqrt{\operatorname{Var}(A)}, E(A)]$ space. The UE frontier is given by two straight lines, the upper one having slope equal to the Sharpe ratio in the previous proposition. Similarly, excess returns on the zero-cost frontier (9) provide zero alphas as a factor when pricing any excess return.

\subsection{UE Returns and Performance Efficient (PE) Returns}

UE returns are defined in Hansen and Richard (1987) as the returns with minimum unconditional variance $\operatorname{Var}(R)$ for each target of unconditional expected return $E(R)$. Hence, the UE returns will be given by the set of returns that solve the problem

$$
\min _{R \in \mathcal{R}} E\left(R^{2}\right) \text { for a given } E(R) \in \mathbb{R}
$$

and we denote these returns by $R_{U}$. Adapting their results to the existence of a safe return, and using the characterization of UE excess returns (10), these UE returns can be represented as

$$
R_{U}=R_{0}\left(1-A^{+}\right)+A_{U}
$$

for some $A_{U}$. These authors proved that the UE returns are a subset of the CE returns, and the representations (8) and (12) reflect this fact. ${ }^{10}$ They also show that UE returns satisfy a beta pricing equation: A return $R_{\beta} \in \mathcal{R}$ different from the minimum unconditional variance one is $\mathrm{UE}$ if and only if, for every $R \in \mathcal{R}$,

$$
E(R)-E_{U}=\frac{\operatorname{Cov}\left(R, R_{\beta}\right)}{\operatorname{Var}\left(R_{\beta}\right)}\left[E\left(R_{\beta}\right)-E_{U}\right]
$$

for some $E_{U} \in \mathbb{R}$. This number is interpreted as the unconditional mean of the corresponding zero-beta return and depends on the chosen UE return factor.

We find that $R_{U}-R_{0}$ cannot be represented as a particular $A_{U}$ in general because its weight on $A^{+}$is random unless $R_{0}$ is constant. Nevertheless, we can define a new type of return efficiency

\footnotetext{
${ }^{10}$ For instance, the safe asset return $R_{0}$ is $\mathrm{CE}$ but it is not UE, while the return $R_{0}\left(1-A^{+}\right)$belongs to both frontiers.
} 
that simply adds $R_{0}$ to a particular $A_{U}$ independently of the randomness of $R_{0}$. Performance efficient (PE) returns solve a slightly different problem with respect to (11),

$$
\min _{R \in \mathcal{R}} E\left[\left(R-R_{0}\right)^{2}\right] \text { for a given } E(R) \in \mathbb{R}
$$

and we denote them by $R_{P}$. The safe asset is riskless with this criterion, which is equivalent to $\operatorname{Var}\left(R-R_{0}\right)$, but is not with $\operatorname{Var}(R)$. PE returns minimize the tracking error with the safe asset as a benchmark. In fact, the investment industry usually measures performance relative to a benchmark and a manager might care about her relative performance more than total risk and return. In this case, we could refer to the excess return $R-R_{0}$ as her active return, and its variance as her tracking error, which is a widely used measure of relative investment risk. See the results of Roll (1992) on tracking error optimization in a context without explicit information neither a safe asset. ${ }^{11}$

Proposition 2 Representation and properties of PE returns defined by (14):

1. PE returns can be represented as

$$
R_{P}=R_{0}+A_{U}
$$

2. The excess returns of $P E$ returns satisfy the same properties in terms of $S_{U}^{2}$ and $\alpha_{U}$ as UE excess returns.

Importantly, adding the safe return to UE excess returns defines a new subset of CE returns (8). This subset, PE returns, has similar properties to UE excess returns. In the case of PE returns, we can find a relationship between their $S_{U}$ and $S$ on the CE frontier,

$$
\frac{1}{1+S_{U}^{2}}=1-E\left(A^{+}\right)=E\left(\frac{1}{1+S^{2}}\right)
$$

This result was already developed by Jagannathan (1996) for UE returns with a constant safe asset return. However, our expressions show that UE returns share the properties of UE excess returns only in the special case of a constant $R_{0}$.

Figure 3 displays the UE and PE frontiers. There is a single optimal risk-return trade-off on the $[\sqrt{\operatorname{Var}(A)}, E(A)]$ space but not on the $[\sqrt{\operatorname{Var}(R)}, E(R)]$ space. The PE frontier is a straight line for positive risk premia on the $[\sqrt{\operatorname{Var}(A)}, E(A)]$ space, and provides the best performance in that space. We can also compare PE and UE frontiers on the $[\sqrt{\operatorname{Var}(R)}, E(R)]$

\footnotetext{
${ }^{11}$ See Section 3 of Peñaranda (2008) for further details on the role of background risks in mean-variance analysis without explicit conditioning information. Note also the differences with respect to Chiang (2009), who is interested in active portfolio management with respect to a general benchmark. Here we want to characterize the subset of CE returns that have maximum unconditional Sharpe ratios and zero unconditional alphas instead.
} 
space, where the latter is the most efficient one. Both frontiers share the location of the minimum and the asymptotes because the only difference between them is the background risk of the safe return. Therefore, these two curves are more different the lower the target return. For comparison, the location of the Markowitz frontier is also shown, interpreted as the PE frontier in (14) but constrained to fixed-weight strategies $\left(R_{0}+\omega \mathbf{A}^{\prime}\left[E\left(\mathbf{A A}^{\prime}\right)\right]^{-1} E(\mathbf{A})\right.$ with $\left.\omega \in \mathbb{R}\right)$ and we refer to these returns as fixed-weight efficient (FE). The FE frontier is also given by a straight line in the left plots, and is very similar to the PE frontier for target returns around the average safe asset. Note that there are regions where fixed-weight portfolios perform better than UE returns.

\section{$<$ Figure 3: UE and PE returns $>$}

The following corollary of Proposition 2 clarifies the difference between UE and PE returns.

Corollary 1 Given the representation of UE and PE returns above,

1. A PE return is related to the UE return with the same mean by

$$
R_{P}=R_{U}+\left[R_{0}-\frac{E\left(R_{0} A^{+}\right)}{E\left(A^{+}\right)}\right] A^{+} .
$$

2. The UE and PE frontiers for returns are equal if and only if

$$
R_{0} \in \mathbb{R}
$$

The right term in point 1 does not depend on the chosen $R_{P}$, has mean zero and is orthogonal to $A^{+}$. It can be interpreted as a hedging demand due to background risk. The difference between both frontiers follows easily from this corollary,

$$
\operatorname{Var}\left(R_{P}\right)=\operatorname{Var}\left(R_{U}\right)+\operatorname{Var}\left(\left[R_{0}-\frac{E\left(R_{0} A^{+}\right)}{E\left(A^{+}\right)}\right] A^{+}\right) .
$$

Therefore, the PE frontier represents a parallel parabola with respect to the UE frontier on the $[\operatorname{Var}(R), E(R)]$ space, where the size of the parallel movement to the right depends on the right term. Point 2 states that the PE and UE frontiers coincide when the safe asset return is constant since then there is no conflict between the mean-variance and hedging motives. In this case, the safe asset return is also UE.

Table 2 shows unconditional Sharpe ratios $S_{U}$ and alphas $\alpha_{U}$ for the different types of efficient returns that we study. The required formulas can be derived from the previous representations. 
Regarding alphas, we could study the pricing of any time-varying strategy of the primitive assets, but we simply focus on pricing the primitive assets. UE returns do not display a constant $S_{U}$ and it may change considerably for target returns around the safe asset. As we increase the target, this $S_{U}$ converges towards the ratio for PE returns, which is its maximum value. Note that the unconditional Sharpe ratio of PE returns is considerably higher than FE returns, and hence there is added value in using conditioning information even in a simple model like ours.

The zero-beta return of UE returns, $E_{U}$ in the beta-pricing equation (13), can be very different from the average safe asset return. UE returns would price any other return with these values of $E_{U}$ and using gross returns in the computation of betas. However, this is not the usual approach in empirical work and $\alpha_{U}$ is more common. For UE returns, $\alpha_{U}$ can be very different from zero and it also depends on the particular target. Here we provide the alphas generated when we use a particular UE return to price one of the primitive excess returns. ${ }^{12}$

$<$ Table 2: Unconditional performance measures $>$

To sum up, when empirical work relies on unconditional Sharpe ratios and Jensen's alphas to test portfolio efficiency is actually testing if a return is PE, not UE. Both efficiencies are only equivalent in the case of a constant safe asset return.

\subsection{Residually Efficient (RE) Excess and Gross Returns}

This section studies a third subset of CE returns. Appendix C analyses the link between mean-variance preferences and frontiers. Ferson and Siegel (2001) show that UE returns can be rationalized by preferences $E(R \mid G)-\left(b_{U} / 2\right) E\left(R^{2} \mid G\right)$ for some strictly positive $b_{U} \in \mathbb{R}$. But UE returns are not related to preferences $E(R \mid G)-\left(\theta_{R} / 2\right) \operatorname{Var}(R \mid G)$ for some strictly positive $\theta_{R} \in \mathbb{R}$, which actually rationalize the efficient returns that we study in this section.

The last preferences only penalize a component of the unconditional variance. We can decompose the unconditional variance of a return as

$$
\operatorname{Var}(R)=E[\operatorname{Var}(R \mid G)]+\operatorname{Var}[E(R \mid G)]
$$

and we refer to the first component as the residual variance because it is the variance of the residual $R-E(R \mid G)$, that is, $\operatorname{Var}(R-E(R \mid G))=E\left[(R-E(R \mid G))^{2}\right]=E[\operatorname{Var}(R \mid G)]$. Note

\footnotetext{
${ }^{12}$ In contrast, fixed-weight portfolios provide zero $\alpha_{U}$ in this application.
} 
that $R_{0}$ is risky from the perspective of $\operatorname{Var}(R)$, but not from the perspective of $\operatorname{Var}(R \mid G)$ and hence $E[\operatorname{Var}(R \mid G)]$.

We define the RE returns as the returns that minimize the residual variance $E[\operatorname{Var}(R \mid G)]$ for a given target of expected return $E(R)$. Thus, the RE returns are given by the set of returns that solve the problem

$$
\min _{R \in \mathcal{R}} E[\operatorname{Var}(R \mid G)] \text { for a given } E(R) \in \mathbb{R}
$$

which we denote by $R_{R}$. Similarly, we denote by $A_{R}$ the excess returns that solve the problem

$$
\min _{A \in \mathcal{A}} E[\operatorname{Var}(A \mid G)] \text { for a given } E(A) \in \mathbb{R} \text {. }
$$

The following proposition studies the properties of RE excess and gross returns. The residual Sharpe ratio of an excess return $A$ is defined as

$$
S_{R}=E(A) / \sqrt{E[\operatorname{Var}(A \mid G)]},
$$

and the residual Jensen's alpha of an excess return $A$ with respect to a pricing factor $A_{\beta}$ is defined as

$$
\alpha_{R}=E(A)-\beta_{R} E\left(A_{\beta}\right), \quad \beta_{R}=E\left[\operatorname{Cov}\left(A, A_{\beta} \mid G\right)\right] / E\left[\operatorname{Var}\left(A_{\beta} \mid G\right)\right] .
$$

Proposition 3 Representation and properties of RE returns defined by (16), and RE excess returns defined by (17):

1. RE excess returns can be represented as

$$
A_{R}=\frac{E\left(A_{R}\right)}{E\left(A^{++}\right)} A^{++} .
$$

2. RE excess returns different from 0 are characterized by achieving the maximum

$$
S_{R}^{2}=E\left(A^{++}\right) .
$$

They are also characterized by

$$
\alpha_{R}=0
$$

when they are used as a factor to price any $A \in \mathcal{A}$.

3. $R E$ returns can be represented as

$$
R_{R}=R_{0}+A_{R}
$$

for some $A_{R}$, and hence their excess returns satisfy the same properties as $R E$ excess returns in terms of $S_{R}^{2}$ and $\alpha_{R}$. 
The RE returns are a subset of the CE returns, as it is the case with UE returns. ${ }^{13}$ The RE frontier is given by two straight lines on the $[\sqrt{E[\operatorname{Var}(R \mid G)]}, E(R)]$ space with zero residual variance at $E(R)=E\left(R_{0}\right)$. In fact, we can see on the left side of Figure 4 that the PE and FE frontiers are also linear in that space. RE returns provide the best risk-return trade-off in that space, while UE returns provide the best frontier on the right side of the plot, which represents $[\sqrt{\operatorname{Var}(R)}, E(R)]$. Note that the safe asset belongs to both the PE and RE frontiers, and hence they are tangent at that point. Interestingly, in some regions, the performance of fixed-weight returns is better than some CE returns.

$<$ Figure 4: UE, PE and RE returns $>$

There is a natural link between the RE frontier and its zero-cost counterpart (17), as it is the case with the $\mathrm{CE}$ and the PE frontiers, but not with the UE frontier. The addition of a safe asset when moving from $\mathcal{A}$ to $\mathcal{R}$ does not change the optimal residual risk-return trade-off, as any $R_{R}$ can be expressed as an $A_{R}$ plus $R_{0}$. There is a single optimal risk-return trade-off on the $[\sqrt{E[\operatorname{Var}(R \mid G)]}, E(R)]$ space in the sense that $S_{R}$ reaches its maximum value for risky returns on the efficient side of the RE. In this regard, there is a simple relationship between $S_{R}$ on the RE frontier and $S$ on the CE frontier

$$
S_{R}^{2}=E\left(A^{++}\right)=E\left(S^{2}\right)
$$

Moreover, every RE return provides $\alpha_{R}=0$ as a factor when pricing any return. This result is the RE counterpart of the beta-pricing characterization of mean-variance frontiers in Roll (1977) for the Markowitz set-up, and Hansen and Richard (1987) for UE returns.

Table 3 illustrates the previous points. RE returns provide the maximum $S_{R}$, whose square is equal to the average square of the conditional Sharpe ratio of CE returns. For instance, using portfolios constructed from MMR, SMB, and HML in Figure 2, we commented that the mean and standard deviation of the conditional Sharpe ratio of CE returns are 0.4118 and 0.7884 respectively. The sum of their squares is equal to the square of 0.8895 , the corresponding value of $S_{R}$ in Table 3. On the other hand, the $S_{R}$ of PE returns lies between the RE and FE returns. These three types of returns display a single value for the residual Sharpe ratio, but this is not

\footnotetext{
${ }^{13}$ For instance, the safe asset return $R_{0}$ is RE but not UE, and the return $R_{0}\left(1-A^{+}\right)$is UE but not RE, while both returns are CE.
} 
the case again for UE returns, which can provide very different values across different target returns. Similarly, there are not pricing errors with RE returns as a pricing factor in terms of $\alpha_{R}$, but this is not the case with UE returns, where once again the particular target matters. Regarding the application of unconditional measures to RE returns, Table 2 showed that the $S_{U}$ for RE returns is constant, and between FE and PE. Similarly, $\alpha_{U}$ are not zero with RE returns, but they do not depend on the particular target.

$<$ Table 3: Residual performance measures $>$

The next corollary characterizes the special cases where the RE is equal to the PE or the UE frontiers: ${ }^{14}$

Corollary 2 Given the representation of RE, UE and PE returns in the previous propositions,

1. The RE and PE frontiers for returns are equal if and only if

$$
E\left(A^{++} \mid G\right) \in \mathbb{R}
$$

that is, the maximum conditional Sharpe ratio is constant. In this case, and only in this case, the RE and UE frontiers for excess returns are equal, and every efficient excess return has a constant conditional mean.

2. The RE and UE frontiers for returns are equal if and only if

$$
R_{0} \in \mathbb{R}, \quad E\left(A^{++} \mid G\right) \in \mathbb{R},
$$

that is, both the safe asset return and the maximum conditional Sharpe ratio are constant. In this case, every efficient return has a constant conditional mean, and these frontiers are also equal to the PE frontier.

Figure 2 showed that these conditions are empirically far from plausible. They are also quite restrictive. In the case of point 2 , the straight lines that represent the $\mathrm{CE}$ frontier on the $[\sqrt{\operatorname{Var}(R \mid G)}, E(R \mid G)]$ space at each value of the conditioning variables in $G$ should be equal.

Figure 5 illustrates the driver of the differences between UE, PE, and RE returns. The three types can be interpreted as different scalings of $A^{+}$, and hence the relative weights across risky assets are the same for these types of efficiency. Figure 5 displays the time series of this scaling for two target returns when the available assets are MMR, SMB, and HML.

\footnotetext{
${ }^{14}$ There are other theoretically possible cases. The RE and the UE frontiers for returns are tangent if and only if there are two real numbers $(a, b)$ such that

$$
R_{0}=a+b E\left(A^{++} \mid G\right)
$$

in which case the shared return has a constant conditional mean $a$. A simple example is $R_{0} \in \mathbb{R}$, since then this return is also UE and hence both frontiers are tangent at $R_{0}$.
} 
$<$ Figure 5: Scaling for efficient returns $>$

The scaling is constant for PE returns, rising from 0.0019 to 0.1424 as the target increases from 5 to $10 \%$, but changes over time for RE and UE returns. As the target increases from 5 to $10 \%$, the average value of the scaling of UE returns increases from 0.0022 to 0.1427 , while the standard deviation stays at 0.0294 . The scaling of RE returns increases in both dimensions, the mean from 0.0016 to 0.1148 , and the standard deviation from 0.0007 to 0.0488 .

\section{Testing Portfolio Efficiency and Asset Pricing Models}

Figure 6 provides a summary of the results in the previous section. In the context of CE excess and gross returns, we have studied two types of efficient excess returns, and three types of efficient gross returns. We can translate RE excess returns into RE gross returns by simply adding the safe asset return. The same operation translates UE excess returns into PE returns. However, the connection between UE excess returns and UE returns is not as straightforward. Now we turn to the implications of our results for testing portfolio efficiency and asset pricing models.

$<$ Figure 6: Summary of efficient excess and gross returns $>$

\subsection{Spanning of Efficient Returns}

Tests of efficiency and asset pricing models are usually stated in terms of excess returns. If we work with an $N \times 1$ vector of excess returns $\mathbf{A}$, which we decompose into an $N_{1} \times 1$ vector $\mathbf{A}_{1}$ and an $N_{2} \times 1$ vector $\mathbf{A}_{2}\left(N=N_{1}+N_{2}\right)$, then the hypothesis of interest is that some portfolio of the elements in $\mathbf{A}_{1}$ lies on the efficient part of the mean-variance frontier spanned by $\mathbf{A}_{1}$ and $\mathbf{A}_{2}$.

From (7), any CE excess return constructed with $\left(\mathbf{A}_{1}, \mathbf{A}_{2}\right)$ is a portfolio of $\mathbf{A}_{1}$ if and only if $A^{+}$has zero weights on $\mathbf{A}_{2}$, or equivalently $A^{++}$has zero weights on $\mathbf{A}_{2}$. From (8), the same condition holds if and only if CE returns with $\left(R_{0}, \mathbf{A}_{1}, \mathbf{A}_{2}\right)$ are constructed with $R_{0}$ and a portfolio of $\mathbf{A}_{1}$. Hence some zero weights in $A^{+}$is the null hypothesis of a conditional efficiency test. This condition is equivalent to some zero conditional alphas because, using the partitioned inverse in (4), we find the equivalent constraints

$$
E\left(\mathbf{A}_{2} \mid G\right)=E\left(\mathbf{A}_{2} \mathbf{A}_{1}^{\prime} \mid G\right)\left[E\left(\mathbf{A}_{1} \mathbf{A}_{1}^{\prime} \mid G\right)\right]^{-1} E\left(\mathbf{A}_{1} \mid G\right)
$$


or

$$
E\left(\mathbf{A}_{2} \mid G\right)=\operatorname{Cov}\left(\mathbf{A}_{2}, \mathbf{A}_{1} \mid G\right)\left[\operatorname{Var}\left(\mathbf{A}_{1} \mid G\right)\right]^{-1} E\left(\mathbf{A}_{1} \mid G\right)
$$

This is a well known result, being an extension to conditional moments of efficiency tests in the Markowitz framework. Our novel result is that the hypothesis to test is the same whatever the particular subset of $\mathrm{CE}$ returns (UE, PE, or RE) we are interested in.

\section{Proposition 4 Spanning of efficient returns:}

1. Any RE or UE excess return constructed with $\left(\mathbf{A}_{1}, \mathbf{A}_{2}\right)$ is a portfolio of $\mathbf{A}_{1}$ if and only if $A^{+}$has zero weights on $\mathbf{A}_{2}$, or equivalently $A^{++}$has zero weights on $\mathbf{A}_{2}$.

2. The same condition holds if and only if $R E, P E$ or $U E$ returns with $\left(R_{0}, \mathbf{A}_{1}, \mathbf{A}_{2}\right)$ are constructed with $R_{0}$ and a portfolio of $\mathbf{A}_{1}$.

The proposition also shows that the hypothesis to test is the same if we study unit cost portfolios instead of zero cost portfolios. All these efficient returns depend on $\mathbf{A}$ only through $A^{+}$or $A^{++}$. Table 1, and Figures 1 and 2, showed already some empirical evidence that is related to this proposition, and to tests of the conditional versions of the CAPM and the Fama-French model. In the case of the CAPM, we test the efficiency of the market portfolio, while in the case of the Fama-French model we test the efficiency of some portfolio of the market, SMB and HML.

In Figure 1, the weights of $A^{+}$and $A^{++}$on HML and WML tend to take higher values than MMR, while SMB usually has the lowest weights. For instance, Table 1 shows that the $A^{+}$ constructed with MMR, SMB, and HML has an average weight on HML twice the average on MMR, while their standard deviations are similar. On the contrary, the weight on SMB has much lower values in both dimensions. Similarly to Lewellen and Nagel (2006), we find that the value premium, represented by HML, is the main source of rejection of the CAPM. ${ }^{15}$ We also find clear evidence that the Fama-French model cannot price WML as its average weight is far from zero. The conditional Sharpe ratio in Figure 2 increases considerably its average level when WML is added. Hence our simple empirical set-up is in line with the empirical literature.

Proposition 4 is a result on both mean-variance efficiency and asset pricing. It provides the conditions under which a portfolio of $\mathbf{A}_{1}$ lies on the mean-variance frontier spanned by $\mathbf{A}$. But the same conditions can be interpreted as $\mathbf{A}_{1}$ defining a set of factors that price the crosssection of assets given by $\mathbf{A}$. For instance, $\mathbf{A}_{1}$ could be a scalar as the market excess return in the CAPM, or a vector that adds size and value factors as in the Fama-French model. In

\footnotetext{
${ }^{15}$ Petkova and Zhang (2005) also conclude that the conditional CAPM cannot explain the value premium.
} 
fact, we can translate the proposition into stochastic discount factors (SDFs). If we define the random variable

$$
m^{*}=\frac{1}{R_{0}} \frac{1-A^{+}}{1-E\left(A^{+} \mid G\right)}=\frac{1}{R_{0}}\left(1-A^{++}+E\left(A^{++} \mid G\right)\right)
$$

then $m^{*}$ satisfies

$$
E\left(m^{*} R_{0} \mid G\right)=1, \quad E\left(m^{*} \mathbf{A} \mid G\right)=\mathbf{0},
$$

which can be interpreted as a proper $\mathrm{SDF}^{16}$ that depends on $\mathbf{A}_{1}$ only. Moreover, note that $1-A^{+}$could also be interpreted as a SDF that prices excess returns,

$$
E\left[\left(1-A^{+}\right) \mathbf{A} \mid G\right]=\mathbf{0},
$$

and similar comments apply to $1-A^{++}+E\left(A^{++} \mid G\right)$ because it satisfies

$$
E\left[\left(1-A^{++}+E\left(A^{++} \mid G\right)\right) \mathbf{A} \mid G\right]=\mathbf{0}
$$

The results in Gallant, Hansen and Tauchen (1990) show that $m^{*}$ is the conditional projection of any random variable $m$ that satisfies the pricing equations (19) onto the conditional span of $R_{0}$ and $\mathbf{A}$. Therefore, we can also use efficiency tests to test asset pricing models with nontraded factors. We can focus on the $m^{*}$ corresponding to a particular model $m$, as the difference $m-m^{*}$ does not play any role in pricing because it is orthogonal to $R_{0}$ and $\mathbf{A}$.

\subsection{Fixed-weight Spanning}

Empirical work often relies on excess returns and SDFs with fixed weights on the pricing factors. In our context, that means

$$
1-A^{+}=1-\lambda_{1}^{\prime} \mathbf{A}_{1}
$$

for some $N_{1} \times 1$ vector $\boldsymbol{\lambda}_{1}$ of real numbers, which can be interpreted as prices of risk. Given a vector of additional excess returns $\mathbf{A}_{2}$, empirical work often relies on the unconditional moments

$$
E\left[\left(\begin{array}{c}
\mathbf{A}_{1} \\
\mathbf{A}_{2}
\end{array}\right)\left(1-\boldsymbol{\lambda}_{1}^{\prime} \mathbf{A}_{1}\right)\right]=\mathbf{0},
$$

which are equivalent to multivariate beta-pricing

$$
E\left(\mathbf{A}_{2}\right)=\operatorname{Cov}\left(\mathbf{A}_{2}, \mathbf{A}_{1}\right)\left[\operatorname{Var}\left(\mathbf{A}_{1}\right)\right]^{-1} E\left(\mathbf{A}_{1}\right) .
$$

\footnotetext{
${ }^{16}$ The duality, or perfect conditional correlation, between conditional frontiers of returns and SDFs is studied in Peñaranda and Sentana (2011a).
} 
For instance, we can test the CAPM with a scalar $\mathbf{A}_{1}$ equal to MMR or test the Fama-French model with a $3 \times 1$ vector $\mathbf{A}_{1}$ (MMR, SMB, and HML).

Similarly, we could work with a fixed-weight SDF

$$
1-A^{++}+E\left(A^{++} \mid G\right)=1-\boldsymbol{\eta}_{1}^{\prime}\left(\mathbf{A}_{1}-E\left(\mathbf{A}_{1} \mid G\right)\right)
$$

for some $N_{1} \times 1$ vector $\boldsymbol{\eta}_{1}$ of real numbers. The corresponding SDF conditions would be

$$
E\left[\left(\begin{array}{c}
\mathbf{A}_{1} \\
\mathbf{A}_{2}
\end{array}\right)\left(1-\boldsymbol{\eta}_{1}^{\prime}\left(\mathbf{A}_{1}-E\left(\mathbf{A}_{1} \mid G\right)\right)\right)\right]=\mathbf{0},
$$

and the corresponding multivariate residual beta-pricing

$$
E\left(\mathbf{A}_{2}\right)=E\left[\operatorname{Cov}\left(\mathbf{A}_{2}, \mathbf{A}_{1} \mid G\right)\right]\left[E\left[\operatorname{Var}\left(\mathbf{A}_{1} \mid G\right)\right]\right]^{-1} E\left(\mathbf{A}_{1}\right) .
$$

The following corollary characterizes the predictability constraints that this type of SDFs impose on top of the zero conditional alphas in Proposition 4.

Corollary 3 Let us assume $A^{+}$, and equivalently $A^{++}$, have zero weights on $\mathbf{A}_{2}$,

1. The weights of $A^{+}$on $\mathbf{A}_{1}$ are constant if and only if there is some $\boldsymbol{\lambda}_{1} \in \mathbb{R}^{N_{1}}$ such that

$$
E\left(\mathbf{A}_{1} \mid G\right)=E\left(\mathbf{A}_{1} \mathbf{A}_{1}^{\prime} \mid G\right) \boldsymbol{\lambda}_{1} .
$$

In this case, PE excess and gross returns are constructed with fixed-weight portfolios of $\mathbf{A}_{1}$, and we can construct a SDF $1-A^{+}$with constant weights on $\mathbf{A}_{1}$.

2. The weights of $A^{++}$on $\mathbf{A}_{1}$ are constant if and only if there is some $\boldsymbol{\eta}_{1} \in \mathbb{R}^{N_{1}}$ such that

$$
E\left(\mathbf{A}_{1} \mid G\right)=\operatorname{Var}\left(\mathbf{A}_{1} \mid G\right) \boldsymbol{\eta}_{1} .
$$

In this case, RE excess and gross returns are constructed with fixed-weight portfolios of $\mathbf{A}_{1}$, and we can construct a SDF $1-A^{++}+E\left(A^{++} \mid G\right)$ with constant weights on $\mathbf{A}_{1}$.

In our simple model of linear risk premia and constant variances, the second condition holds if and only if there is no predictability in $\mathbf{A}_{1}$. That is, the predictive regression slopes are zero. This fact suggests that these conditions may not hold in usual models in empirical finance, even though return predictability and these conditions could coexist in general.

The case of $N_{1}=1$ is specially interesting as it is connected to the efficiency of a particular portfolio, and hence to the CAPM when that portfolio is the market return. The second condition means $E\left(A_{1} \mid G\right) / \operatorname{Var}\left(A_{1} \mid G\right) \in \mathbb{R}$ in that case, and then $A_{1}$ is $\mathrm{RE}$ and $R_{1}=A_{1}+R_{0}$ is also RE. That is, the condition means that the particular portfolio itself is RE, which does not need to be the case under zero conditional alphas only. Similarly, the first condition means that $E\left(A_{1} \mid G\right) / E\left(A^{2} \mid G\right) \in \mathbb{R}$, and then $A_{1}$ is UE and $R_{1}=A_{1}+R_{0}$ is PE. However, $R_{1}=A_{1}+R_{0}$ is 
UE if and only if $R_{0}+E\left(A_{1}^{2} \mid G\right) / E\left(A_{1} \mid G\right) \in \mathbb{R}$ instead. Ferson and Siegel (2009) propose a test of unconditional efficiency based on facing the unconditional Sharpe ratio of a portfolio against the maximum one. As commented before, such a test should be interpreted as the corresponding portfolio excess return being UE or the portfolio return being PE. Furthermore, now we see the implied predictability constraints in such a test, as we are imposing that the portfolio itself lies on a particular frontier.

Under the conditions of Proposition 4, a single excess return $A_{1}$ will be CE but not necessarily $\mathrm{UE}, \mathrm{PE}$ or $\mathrm{RE}$, since in general $A^{+}$or $A^{++}$are equal to a conditional rescaling of $A_{1}$. For $A_{1}$ to be UE, PE or RE, additional predictability constraints would be required such that optimal excess returns do not use information. Let us briefly study this point with RE returns, which can be represented as

$$
R_{R}=R_{0}+w_{R} A^{++}=R_{0}+w_{R} \frac{E\left(A_{1} \mid G\right)}{\operatorname{Var}\left(A_{1} \mid G\right)} A_{1}, \quad w_{R} \in \mathbb{R}
$$

$R_{1}$ will not be on the RE frontier unless there is a $\dot{w}_{R}$ such that

$$
\dot{w}_{R} \frac{E\left(A_{1} \mid G\right)}{\operatorname{Var}\left(A_{1} \mid G\right)}=1
$$

which requires that $E\left(A_{1} \mid G\right) / \operatorname{Var}\left(A_{1} \mid G\right)$ is a real number. Moreover, this condition would imply that RE returns are fixed-weight portfolios of $R_{1}$ and $R_{0}$, and hence these optimal returns would not use conditioning information.

A classic example of efficiency tests is testing the validity of the CAPM, a model where the market portfolio must be mean-variance efficient in equilibrium. ${ }^{17}$ The conditional CAPM assumes that investors choose CE returns. If the safe asset is in zero net supply, as it is usually assumed, then the market return is only composed of risky returns. In that case, in equilibrium the market return, say $R_{1}$ in the previous paragraphs, is actually CE. However, the results above show that such a return is not RE, PE or UE unless returns satisfy some predictability constraints. In particular, if we think of mean-variance preferences such that agents' returns and their fixed-weight aggregation are RE, and a safe asset in zero net-supply, then equilibrium imposes a constant $\operatorname{Var}\left(A_{1} \mid G\right) / E\left(A_{1} \mid G\right)$ and optimal portfolio weights are independent of information. These results are related to the existence of a tangency portfolio, which is studied in Appendix D.

\footnotetext{
${ }^{17}$ The model was originally developed by Sharpe (1964), Lintner (1965) and Mossin (1966) in a context without conditioning information.
} 
We commented that Panel $\mathrm{C}$ of Table 1 shows that weights of $A^{+}$and $A^{++}$have standard deviations of similar order compared to their means, that is, there is considerable time-variation in these weights. Now we can interpret this empirical fact as rendering SDFs with fixed weights invalid to price time-varying strategies. Similarly, we can see graphically the relevant timevariation in the MMR weight in the first row of Figure 1, which invalidates a CAPM-like SDF with a fixed weight on the market return. We can also see the time-variation of weights on the three Fama-French factors in the second row, and hence the same comments apply to a Fama-French SDF with fixed-wights.

\section{Conclusions and Further Research}

Our results can be considered as a guideline for future empirical work, and an accurate interpretation of the current evidence, on testing mean-variance efficiency and asset pricing models. Our main contribution is a careful analysis of three different types of efficient returns in the presence of conditioning information, as summarized in Figure 6.

We prove that unconditionally efficient returns do not show a unique optimal unconditional Sharpe ratio, and their unconditional Jensen's alphas are not zero either, even though these measures are widely used in empirical finance. This fact opens the question of what type of efficiency is actually tested in empirical finance, and next we characterize a new set of efficient returns that actually satisfies those unconditional properties. We also characterize a third set of efficient returns that is rationalized by standard mean-variance preferences and motivates new Sharpe ratios and Jensen's alphas.

We conclude our theoretical analysis by showing that, when there is portfolio of a set of returns that lies on the mean-variance frontier spanned by an extended set of returns, any of the three types of efficiency requires zero conditional alphas, and hence there is a unique null hypothesis to test. However, if we think of the CAPM for instance, and we also want to impose that the market return itself is efficient in a particular sense then the null hypothesis adds predictability constraints of different types on the market return.

Our empirical application revisits the conditional variants of the CAPM and the Fama-French model. We use four excess returns that capture the market, and the size, value, and momentum effects, and consider three prominent predictors. We find return predictability in these data, with considerable time-variation in the weights of efficient returns. This fact invalidates asset 
pricing models with fixed-weight stochastic discount factors. The value and momentum effects are far more relevant than the size effect, and also their weights in optimal portfolios tend to be higher than the weights on the market return. We find that the value effect is more important than the size effect as a source of rejection of the CAPM, and we also find that the Fama-French model cannot price momentum.

The unconditional Sharpe ratio of UE returns changes considerably for target returns around the safe asset. As we increase the target, this Sharpe ratio converges towards the ratio for $\mathrm{PE}$ returns, which is its maximum value. The unconditional Sharpe ratio of PE returns is considerably higher than the ratio we can achieve with fixed-weight returns, which reflects the added value in the conditioning information. Nevertheless, there are regions in mean-variance spaces where fixed-weight portfolios perform better than some CE returns. The zero-beta return of UE returns can be very different from the average return on the safe asset. However, this is not the usual approach in empirical work, where using excess returns in the computations of unconditional alphas is more common. For UE returns, these alphas can be very different from zero and also depend on the particular target return.

There are some interesting avenues for further research. We could analyze the new types of efficient returns in terms of portfolio performance, as e.g. Chiang (2009) does for UE returns with a benchmark, or Avramov and Chordia (2006) do in a real-time Bayesian set-up. In fact, our analysis has provided new efficiency measures that may be useful in performance evaluation. Similarly, we could use the new efficient returns to compute factor mimicking portfolios, as Ferson, Siegel, and Xu (2006) have computed such portfolios with UE returns. The analysis of more general preferences that include higher order moments and intertemporal efficiency are additional topics of further research.

As it is common in empirical work, the efficiency issues that we have studied focus on the use of a safe asset and excess returns. Nevertheless, Appendix D studies the relationship between frontiers with and without a safe asset. If such an asset is not available (e.g. the investor does not have access to a safe asset in real terms) then we can think of testing spanning or tangency. These tests are studied in Peñaranda and Sentana (2010, 2011b) in a framework that does not take into account information explicitly, and we plan to develop the corresponding extensions.

Finally, our empirical application served as a standard illustration of our theoretical concepts, and hence we were not specially concerned about the potential misspecification of the conditional 
moments. Testing zero conditional alphas seems to require the correct model of first and second conditional moments of asset returns, and Ghysels (1998) stresses the impact of misspecification in this context. In addition, a simple model as the one we used may not be rich enough to explore interesting situations. However, as Hansen and Richard (1987) already pointed out, there is no loss of information in moving from conditional to unconditional moments, but only as long as we consider all zero-cost portfolio strategies, and not simply a subset (e.g. fixed-weight portfolios). In fact, managed portfolios usually approximate portfolio strategies by linear functions of the predictors, but they can provide the basis for a proper non-parametric procedure. ${ }^{18}$ Sieve methods can approximate any portfolio strategy whose weights are a smooth function of the predictors, and we plan to study this non-parametric approach in subsequent research. See Chen (2007) for a survey of sieves methods.

\footnotetext{
${ }^{18}$ There are also other non-parametric approaches. Wang (2003) estimates the weights of the SDF as nonparametric functions of the predictors. Lewellen and Nagel (2006) rely on short-window regressions to estimate conditional moments without the use of predictors.
} 


\section{References}

Avramov, D., and T. Chordia (2006): "Predicting Stock Returns", Journal of Financial Economics 82, 387-415.

Bansal, R., M. Dahlquist, and C.R. Harvey (2004): "Dynamic Strategies and Portfolio Choice", NBER WP 10820.

Basak, S., and G. Chabakauri (2010): "Dynamic Mean-Variance Asset Allocation", Review of Financial Studies 23, 2970-3016.

Brandt, M.W., and P. Santa-Clara (2006): "Dynamic Portfolio Selection by Augmenting the Asset Space", Journal of Finance 61, 2187-2217.

Brunnermeier, M.K. (2001): "Asset Pricing under Asymmetric Information", Oxford University Press.

Chamberlain, G. (1983): "A Characterization of the Distributions that Imply Mean-Variance Utility Functions", Journal of Economic Theory 29, 185-201.

Chamberlain, G. and M. Rothschild (1983): “Arbitrage, Factor Structure, and Mean-Variance Analysis on Large Asset Markets", Econometrica 51, 1281-1304.

Chen, X. (2007): "Large sample sieve estimation of semi-nonparametric models", in J. Heckman and E. Leamer (eds.), Handbook of Econometrics Vol. VI, Elsevier.

Chiang, I-H. E. (2009): "Modern Portfolio Management with Conditioning Information", mimeo.

Cochrane, J.H. (2001): “Asset Pricing”, Princeton University Press.

Dybvig, P.H., and S.A. Ross (1985): "Differential Information and Performance Measurement Using a Security Market Line", Journal of Finance 40, 383-399.

Easly, D., and M. O'Hara (2004): "Information and the Cost of Capital", Journal of Finance $59,1553-1583$.

Engle, R. (2002): "Dynamic Conditional Correlation: A Simple Class of Multivariate Generalized Autoregressive Conditional Heteroskedasticity Models", Journal of Business and Economic Statistics 20, 339-350.

Fama, E.F., and K.R. French (1993): "Common Risk Factors in the Returns on Stock and Bonds", Journal of Financial Economics 33, 3-56.

Ferson, W.E., and A.F. Siegel (2001): "The Efficient Use of Conditioning Information in Portfolios", Journal of Finance 56, 967-982. 
Ferson, W.E., and A.F. Siegel (2009): "Testing Portfolio Efficiency with Conditioning Information", Review of Financial Studies 22, 2735-2758.

Ferson, W.E., A.F. Siegel, and P. Xu (2006): "Mimicking Portfolios with Conditioning Information", Journal of Financial and Quantitative Analysis 41, 607-636.

Gallant, A.R., L.P. Hansen and G. Tauchen (1990): "Using Conditional Moments of Asset Payoffs to Infer the Volatility of Intertemporal Marginal Rates of Substitution", Journal of Econometrics 45, 141-179.

Ghysels, E. (1998): "On Stable Factor Structures in the Pricing of Risk: Do Time-Varying Betas Help or Hurt?", Journal of Finance 53, 549-573.

Gibbons, M.R., S.A. Ross, and J. Shanken (1989): "A Test of the Efficiency of a Given Portfolio", Econometrica 57, 1121-1152.

Hansen, L.P., and S.F. Richard (1987): "The Role of Conditioning Information in Deducing Testable Restrictions Implied by Dynamic Asset Pricing Models", Econometrica 55, 587-613.

Jagannathan, R. (1996): "Relation between the Slopes of the Conditional and Unconditional Mean-Standard Deviation Frontiers of Asset Returns", in S. Saito, K. Sawaki, and K. Kubota (eds.) Modern Portfolio Theory and its Applications, Center for Academic Societies, Osaka.

Jegadeesh, N., and Titman, S. (1993): "Returns to Buying Winners and Selling Losers: Implications for Stock Market Efficiency", Journal of Finance 48, 65-91.

Lewellen, J. and S. Nagel (2006): "The Conditional CAPM Does Not Explain Asset-Pricing Anomalies", Journal of Financial Economics 82, 289-314.

Lintner, J. (1965): "The Valuation of Risk Assets and the Selection of Risky Investments in Stock Portfolios and Capital Budgets", Review of Economics and Statistics 47, 13-37.

Markowitz, H. (1952): "Portfolio Selection", Journal of Finance 7, 77-99.

Mossin, J. (1966): "Equilibrium in a Capital Asset Market", Econometrica 34, 768-783.

Owen, J., and R. Rabinovitch (1983): "On the Class of Elliptical Distributions and their Applications to the Theory of Portfolio Choice", Journal of Finance 58, 745-752.

Peñaranda, F. (2008): "Portfolio Choice Beyond the Traditional Approach", Revista de Economía Financiera (now Spanish Review of Financial Economics), 15, 50-90.

Peñaranda, F. and E. Sentana (2010): "Spanning Tests in Portfolio and Stochastic Discount Factor Mean-Variance Frontiers: A Unifying Approach", forthcoming in Journal of Econometrics. 
Peñaranda, F., and E. Sentana (2011a): "Duality in Mean-Variance Frontiers with Conditioning Information", revised version of UPF WP 1058.

Peñaranda, F. and E. Sentana (2011b): "Inferences about Portfolio and Stochastic Discount Factor Mean-Variance Frontiers", mimeo, UPF.

Petkova, R., and L. Zhang (2005): "Is Value Riskier than Growth?", Journal of Financial Economics 78, 187-202.

Roll, R. (1977): "A Critique of the Asset Pricing Theory's Tests Part I: On Past and Potential Testability of the Theory", Journal of Financial Economics 4, 129-176.

Roll, R. (1992): A Mean-Variance Analysis of Tracking Error, Journal of Portfolio Management 18, Summer.

Schwert, G.W. (2003): "Anomalies and Market Efficiency", in G.M. Constantinides, M. Harris, and R. Stulz, Handbook in Economic and Finance.

Sentana, E. (2009): "The Econometrics of Mean-Variance Efficiency Tests: A Survey", Econometrics Journal 12, C65-C101.

Sharpe, W.F. (1964): "Capital Asset Prices: A Theory of Market Equilibrium under Conditions of Risk", Journal of Finance 19, 425-442.

Wang, K.Q. (2003): “Asset Pricing with Conditioning Information: A New Test", Journal of Finance 58, 161-196. 


\section{Appendix}

\section{A Proofs}

The proofs below are based on several types of orthogonal projections. We assume that the diagonal elements of $E\left(\mathbf{A A}^{\prime} \mid G\right)$ in (1) are uniformly bounded with probability one, so that all the elements of $\mathbf{A}$ belong to $L^{2}$, which is the collection of all random variables defined on the underlying probability space with bounded unconditional second moments.

\section{Proposition 1:}

1) We can decompose any $A \in \mathcal{A}$ into two components that belong to $\mathcal{A}$

$$
A=\eta A^{+}+u, \quad \eta=\frac{E\left(A^{+} A\right)}{E\left(A^{+2}\right)}=\frac{E(A)}{E\left(A^{+}\right)}
$$

where the first component is the unconditional projection of $A$ onto the unconditional span of $A^{+}$, and $u$ is the projection error. The error satisfies $E(u)=0$ because $E\left(A^{+} u\right)=0$.

Therefore, the error does not affect $E(A)$ but increases $E\left(A^{2}\right)$. The excess returns that solve problem (9) cannot have an error term, and hence the solution can be represented as

$$
A_{U}=\eta A^{+}, \quad \eta=\frac{E\left(A_{U}\right)}{E\left(A^{+}\right)} .
$$

2) When $E\left(A_{U}\right) \neq 0$, the $S_{U}^{2}$ of those optimal excess returns is given by

$$
\frac{E^{2}\left(A_{U}\right)}{\operatorname{Var}\left(A_{U}\right)}=\frac{\eta^{2} E^{2}\left(A^{+}\right)}{\eta^{2} E\left(A^{+}\right)\left(1-E\left(A^{+}\right)\right)}=\frac{E\left(A^{+}\right)}{1-E\left(A^{+}\right)},
$$

and, as a pricing factor, they provide $\alpha_{U}=0$ for any $A \in \mathcal{A}$ because

$$
\frac{\operatorname{Cov}\left(A, A_{U}\right)}{\operatorname{Var}\left(A_{U}\right)} E\left(A_{U}\right)=\frac{\eta E(A)\left(1-E\left(A^{+}\right)\right)}{\eta^{2} E\left(A^{+}\right)\left(1-E\left(A^{+}\right)\right)} \eta E\left(A^{+}\right)=E(A) .
$$

On the other hand, any risky $A_{\beta}$ such that

$$
\frac{E\left(A^{+}\right)}{1-E\left(A^{+}\right)}=\frac{E^{2}\left(A_{\beta}\right)}{\operatorname{Var}\left(A_{\beta}\right)}
$$

or such that

$$
E(A)=\frac{\operatorname{Cov}\left(A, A_{\beta}\right)}{\operatorname{Var}\left(A_{\beta}\right)} E\left(A_{\beta}\right)
$$

for any $A \in \mathcal{A}$, must be equal to a particular $A_{U}$. The reason is that we can decompose this $A_{\beta}$ as an underlying $A_{U}$ plus an error $u$ from the decomposition of excess returns above. Thus the Sharpe ratio of $A_{\beta}$ satisfies

$$
\frac{E^{2}\left(A_{U}\right)}{\operatorname{Var}\left(A_{U}\right)}=\frac{E^{2}\left(A_{U}\right)}{\operatorname{Var}\left(A_{U}\right)+\operatorname{Var}(u)}
$$


and similarly, pricing the underlying $A_{U}, A_{\beta}$ satisfies the beta-pricing equation

$$
E\left(A_{U}\right)=\frac{\operatorname{Var}\left(A_{U}\right)}{\operatorname{Var}\left(A_{U}\right)+\operatorname{Var}(u)} E\left(A_{U}\right)
$$

Any of the previous conditions implies that $\operatorname{Var}(u)=0$, which translates into $u=E(u)=0$.

\section{Proposition 2:}

1) For any $R \in \mathcal{R}$, we can decompose $R-R_{0} \in \mathcal{A}$ in a similar way to $A$ in the proof of point 1 in Proposition 1, and show that PE returns can be represented as

$$
R_{P}=R_{0}+\omega_{P} A^{+} \quad \omega_{P} \in \mathbb{R}
$$

By choosing the $A_{U}$ associated to $E\left(A_{U}\right) / E\left(A^{+}\right)=\omega_{P}$,

$$
R_{P}=R_{0}+\omega_{P} A^{+}=R_{0}+A_{U}
$$

and hence $R_{P}-R_{0}$ can be represented as a particular $A_{U}$.

2) We can use the proof of point 2 in Proposition 1 with $A_{U}$ and $A_{\beta}$ equal to $R_{P}-R_{0}$.

\section{Proposition 3:}

1) This proof relies on the residual inner product $E[\operatorname{Cov}(x, y \mid G)]$ between random variables $x$ and $y$ and its corresponding norm as $\sqrt{E[\operatorname{Var}(x \mid G)]}$. A priori, this may not be a proper norm in the sense that $\operatorname{Var}(x \mid G)=0$ implies $x=E(x \mid G)$ but not necessarily $x=0$. However, if there is not a safe asset, i.e. there is no $R$ such that $R=E(R \mid G) \neq 0$, then $\operatorname{Var}(R \mid G)=0$ implies $R=0$. Moreover, even if there was a safe asset, this inner product would define a proper norm in $\mathcal{A}$ if there are no arbitrage opportunities, because a safe asset cannot belong to $\mathcal{A}$.

We can decompose any $A \in \mathcal{A}$ into two components that belong to $\mathcal{A}$

$$
A=\lambda A^{++}+u, \quad \lambda=\frac{E\left[\operatorname{Cov}\left(A, A^{++} \mid G\right)\right]}{E\left[\operatorname{Var}\left(A^{++} \mid G\right)\right]}=\frac{E(A)}{E\left(A^{++}\right)},
$$

where the first component is the residual projection of $A$ onto the unconditional span of $A^{++}$, and $u$ is the projection error. The error satisfies $E(u)=0$ because $E\left[\operatorname{Cov}\left(A^{++}, u \mid G\right)\right]=0$.

The error does not affect $E(A)$ but increases $E[\operatorname{Var}(A \mid G)]$ and hence optimal excess returns cannot have an error term. The solution of problem (17) is

$$
A_{R}=\lambda A^{++}, \quad \lambda=\frac{E\left(A_{R}\right)}{E\left(A^{++}\right)} .
$$


2) When $E\left(A_{R}\right) \neq 0$, the $S_{R}^{2}$ of these optimal excess returns is given by

$$
\frac{E^{2}\left(A_{R}\right)}{E\left[\operatorname{Var}\left(A_{R} \mid G\right)\right]}=\frac{\lambda^{2} E^{2}\left(A^{++}\right)}{\lambda^{2} E\left(A^{++}\right)}=E\left(A^{++}\right)
$$

and, as a pricing factor, they provide $\alpha_{R}=0$ for any $A \in \mathcal{A}$ because

$$
\frac{E\left[\operatorname{Cov}\left(A, A_{R} \mid G\right)\right]}{E\left[\operatorname{Var}\left(A_{R} \mid G\right)\right]} E\left(A_{R}\right)=\frac{\lambda E(A)}{\lambda^{2} E\left(A^{++}\right)} \lambda E\left(A^{++}\right)=E(A) .
$$

On the other hand, any risky $A_{\beta}$ such that

$$
E\left(A_{0}^{++}\right)=\frac{E^{2}\left(A_{\beta}\right)}{E\left[\operatorname{Var}\left(A_{\beta} \mid G\right)\right]}
$$

or such that

$$
E(A)=\frac{E\left[\operatorname{Cov}\left(A, A_{\beta} \mid G\right)\right]}{E\left[\operatorname{Var}\left(A_{\beta} \mid G\right)\right]} E\left(A_{\beta}\right)
$$

for any $A \in \mathcal{A}$, must be equal to a particular $A_{R}$. We can decompose this $A_{\beta}$ as an underlying $A_{R}$ plus some error, and show that the conditions above imply that the error must be zero.

3) For any $R \in \mathcal{R}$, we can decompose $R-R_{0} \in \mathcal{A}$ in a similar way to $A$ in the proof of point 1 , and show that RE returns can be represented as

$$
R_{R}=R_{0}+\omega_{R} A^{++} \quad \omega_{R} \in \mathbb{R} .
$$

Finally, by choosing the $A_{R}$ associated to $\lambda=\omega_{R}$,

$$
R_{R}=R_{0}+\omega_{R} A^{++}=R_{0}+A_{R}
$$

and hence $R_{R}-R_{0}$ can be represented as a particular $A_{R}$.

\section{Proposition 4:}

1) Any UE excess return is equal to $A^{+}$times a real number, and $A^{+}$is a portfolio of $\left(\mathbf{A}_{1}, \mathbf{A}_{2}\right)$. Therefore UE excess returns do not take position in $\mathbf{A}_{2}$ if and only if $A^{+}$does not. A similar argument applies to RE excess and $A^{++}$. Finally, $A^{+}$and $A^{++}$are conditionally proportional to each other, so one of them does not take position in $\mathbf{A}_{2}$ if and only if the other does not either.

2) RE, PE or UE returns with $\left(R_{0}, \mathbf{A}_{1}, \mathbf{A}_{2}\right)$ are constructed as $R_{0}$ plus $A^{+}$times a random variable in $I$, which is actually a real number in the case of PE returns. Therefore, we can follow the same argument as in point 1. 


\section{B Monthly Data}

In the case of monthly data, we model $E(\mathbf{A} \mid G)$ as linear in the predictors, and $\operatorname{Var}(\mathbf{A} \mid G)$ is constructed by means of the Dynamic Conditional Correlation (DCC) methodology of Engle (2002). In this model, the relevant information for risk premia is given by the three predictors, while the relevant information for variances is given by past returns. Below we report results for two sets of excess returns, one with MMR, SMB, and HML, and another one that adds WML.

Table A1 shows some annualized descriptive statistics, the outcome of predictive regressions with monthly data, and the corresponding weights of the representing portfolios. As it is well known, the $R^{2}$ in monthly predictive regressions of MMR is much lower than in annual regressions. Here note also the big drop for WML. In any case, the Wald test still rejects predictability for MMR, SMB, and jointly. The weights of the representing portfolios show averages of a similar order to their standard deviations again. The average weights of $A^{+}$are similar to its fixed-weight counterpart.

$<$ Table A1: Description of monthly data $>$

The annualized mean of the safe asset return is 4.7877 , not far from its counterpart with annual data, but its annualized standard deviation is much lower, 0.8306. This is due to the high persistence in interest rates. Regarding the behavior of the annualized conditional Sharpe ratio of $\mathrm{CE}$ returns, it has a higher mean and standard deviation than the annual figures. In particular, with MMR, SMB, and HML we can achieve an average ratio of 1.0817 with a standard deviation of 0.4804 . If add WML then the average increases to 1.6755 and the standard deviation to 0.6021 .

$<$ Table A2: Unconditional performance measures $>$

Tables A2 and A3 show that the annual patterns still hold at the monthly frequency, albeit they are less pronounced.

$<$ Table A3: Residual performance measures $>$ 


\section{Preferences Underlying UE and RE Returns}

In the classic Markowitz set-up based on fixed-weight portfolios, any family of mean-variance preferences can be chosen to explore the whole efficient mean-variance frontier. For instance, the passive returns that maximize $E(R)-(b / 2) E\left(R^{2}\right)$ for each real number $b$ lie on the meanvariance frontier, and each of those optimal returns also maximizes $E(R)-(\theta / 2) \operatorname{Var}(R)$ for the corresponding real number $\theta$. This is not the case when we take into account that investors design portfolio strategies given a nontrivial information set $G$.

The justification of mean-variance preferences under the expected utility paradigm was linked to elliptical distributions by Chamberlain (1983) and Owen and Rabinovitch (1983) in the

Markowitz set-up. Mean-variance preferences can be represented by $U(E(R \mid G), \sqrt{\operatorname{Var}(R \mid G)})$ for some function $U(\cdot)$ that may depend on $G$ and satisfies some standard properties: strictly increasing in the first argument, strictly decreasing in the second argument, and strictly concave in both. These preferences rationalize CE returns.

We can map mean-variance preferences, or equivalently the CE problem (5), into simple criteria based on possibly random risk-return trade-offs. The following mean-variance criteria rationalize CE returns (the corresponding proof is available upon request from the author):

1. The optimal return of problem

$$
\max _{R \in \mathcal{R}} E(R \mid G)-\frac{\theta}{2} \operatorname{Var}(R \mid G),
$$

given some strictly positive $\theta \in I$ is equal to (8) for $E(R \mid G) \in I$ such that

$$
\frac{E(R \mid G)-R_{0}}{E\left(A^{++} \mid G\right)}=\frac{1}{\theta}
$$

2. The optimal return of problem

$$
\max _{R \in \mathcal{R}} E(R \mid G)-\frac{b}{2} E\left(R^{2} \mid G\right)
$$

given some strictly positive $b \in I$ is equal to (8) for $E(R \mid G) \in I$ such that

$$
\frac{E(R \mid G)-R_{0}}{E\left(A^{+} \mid G\right)}=\frac{1}{b}-R_{0}
$$

Ferson and Siegel (2001) show that the optimal portfolio of an agent with quadratic utility $E\left[R-\left(b_{U} / 2\right) R^{2} \mid G\right]$ for some strictly positive $b_{U} \in \mathbb{R}$ is actually an UE return. In our set-up, 
the result above confirms that the solution of problem

$$
\max _{R \in \mathcal{R}} E(R \mid G)-\frac{b_{U}}{2} E\left(R^{2} \mid G\right)
$$

is equal to the solution of problem (11) for the target $E(R) \in \mathbb{R}$ corresponding to the unconditional mean of the return

$$
R_{0}+\left(\frac{1}{b_{U}}-R_{0}\right) A^{+}
$$

Nevertheless, when conditioning information is taken into account, probably the most common mean-variance preferences in finance theory are given by

$$
\max _{R \in \mathcal{R}} E(R \mid G)-\frac{\theta_{R}}{2} \operatorname{Var}(R \mid G) \text {. }
$$

for some strictly positive $\theta_{R} \in \mathbb{R}$. Areas such as market microstructure and rational expectations equilibria often rely on those preferences, see e.g. Brunnermeier (2001) for a survey of asset pricing theory under asymmetric information or Easly and O'Hara (2004) as a more recent reference. They are also used in continuous time asset allocation, see Basak and Chabakauri (2010) and the references therein. These preferences are also used in Dybvig and Ross (1985) to study the complexity of performance evaluation of an informed manager by an uninformed agent. The criterion (C2) is often justified by CARA utility $E\left[-\exp \left(-\theta_{R} R\right) \mid G\right]$ plus conditional normality of $R$, but none of our results require CARA utility and/or normality.

Given the first point above, we can easily characterize the specific CE subset where the choices of this criterion are located. The optimal return that solves problem $(\mathrm{C} 2)$ is

$$
R_{0}+\frac{1}{\theta_{R}} A^{++}
$$

and the RE frontier is the mean-variance frontier where only these returns are located.

\section{The Tangency Portfolio for UE and RE Returns}

Most of the paper studies the case where there is a safe asset. But here we describe the more general context where such an asset may not be available, and study the connection between both cases. Let us briefly review the general case where the space of active returns $\mathcal{R}$ may not include a safe asset, as originally studied in Hansen and Richard (1987). We define $R^{*}$ (the return with minimum conditional second moment $\left.E\left(R^{2} \mid G\right)\right)$ and $\mathfrak{A}^{+}$as the counterparts of $R_{0}\left(1-A^{+}\right)$ and $A^{+}$respectively. Chamberlain and Rothschild (1983) developed a representation of the Markowitz frontier based on an alternative pair of payoffs. In our set-up, we define $R^{* *}$ (the 
return with minimum conditional variance $\operatorname{Var}(R \mid G))$ and $\mathfrak{A}^{++}$as the counterparts of $R_{0}$ and $A^{++}$respectively.

Using this notation, the CE returns can be represented as

$$
R_{C}=R^{*}+\frac{E\left(R_{C}-R^{*} \mid G\right)}{E\left(\mathfrak{A}^{+} \mid G\right)} \mathfrak{A}^{+}=R^{* *}+\frac{E\left(R_{C}-R^{* *} \mid G\right)}{E\left(\mathfrak{A}^{++} \mid G\right)} \mathfrak{A}^{++},
$$

the UE returns can be represented as

$$
R_{U}=R^{*}+\frac{E\left(R_{U}-R^{*}\right)}{E\left(\mathfrak{A}^{+}\right)} \mathfrak{A}^{+}
$$

and we can also extend our representation of RE returns to this set-up,

$$
R_{R}=R^{* *}+\frac{E\left(R_{R}-R^{* *}\right)}{E\left(\mathfrak{A}^{++}\right)} \mathfrak{A}^{++} .
$$

Moreover, we can extend the beta-pricing results that we studied to the case where a safe asset may not be available. The beta-pricing statement is: A return $R_{\beta} \in \mathcal{R}$ different from the minimum variance one is $\mathrm{CE}$ if and only if, for every $R \in \mathcal{R}, E(R \mid G)-E=$ $\left[\operatorname{Cov}\left(R, R_{\beta} \mid G\right) / \operatorname{Var}\left(R_{\beta} \mid G\right)\right]\left[E\left(R_{\beta} \mid G\right)-E\right]$ for some $E \in I$. That random variable is interpreted as the conditional mean of the corresponding zero-beta return. It is unique and equal to the safe asset return if such an asset exists. Similarly, a return $R_{\beta} \in \mathcal{R}$ different from the minimum unconditional variance one is UE if and only if, for every $R \in \mathcal{R}$, $E(R)-E_{U}=\left[\operatorname{Cov}\left(R, R_{\beta}\right) / \operatorname{Var}\left(R_{\beta}\right)\right]\left[E\left(R_{\beta}\right)-E_{U}\right]$ for some $E_{U} \in \mathbb{R}$. This number is interpreted as the unconditional mean of the corresponding zero-beta return and depends on the chosen UE return factor. Conditional and unconditional beta-pricing were studied in Hansen and Richard (1987), and hence our contribution in this respect is that there is similar beta-pricing equation for RE returns if we use $E\left[\operatorname{Cov}\left(R, R_{\beta} \mid G\right)\right] / E\left[\operatorname{Var}\left(R_{\beta} \mid G\right)\right]$ as beta.

The CE frontier without a safe asset is a hyperbola on the $[\sqrt{\operatorname{Var}(R \mid G)}, E(R \mid G)]$ space for a particular value of the conditioning variables in $G$. In general there is a conditional mean $E(R \mid G)$ such that the weight of the CE return on the conditionally safe payoff will be identically 0 for every possible signal realization. This unique optimal return that is shared by the CE frontier with and without a safe asset is called the tangency portfolio. The only exception where a tangency does not exist is the case $E\left(R^{* *} \mid G\right)=R_{0}$ because then the cost of the risky component of the CE frontier with a safe asset would be zero at every $E(R \mid G)$.

The risky component of the CE returns with a safe asset is conditionally proportional to the tangency return, which we characterize in the following result (the corresponding proof is 
available upon request from the author): If $E\left(R^{* *} \mid G\right) \neq R_{0}$ then there is a tangency portfolio between the CE frontier with and without a safe asset given by

$$
R^{* *}+\frac{\operatorname{Var}\left(R^{* *} \mid G\right)}{E\left(R^{* *} \mid G\right)-R_{0}} \mathfrak{A}^{++}=R^{*}+\left[\frac{E\left(R^{* 2} \mid G\right)-R_{0} E\left(R^{*} \mid G\right)}{E\left(R^{*} \mid G\right)-R_{0}\left(1-E\left(\mathfrak{A}^{+} \mid G\right)\right)}\right] \mathfrak{A}^{+},
$$

which is not RE or UE in general.

The UE and the RE frontiers without a safe asset are hyperbolas on their respective spaces. In contrast with the textbook treatment of mean-variance frontiers, in general there is no tangency in any of them. Peñaranda and Sentana (2011a) already pointed out this fact for the UE frontier. We can characterize the special cases where we can find a tangency on the RE or the UE frontiers by means of the previous result. Let us assume $E\left(R^{* *} \mid G\right) \neq R_{0}$ :

1. The CE tangency portfolio is RE when

$$
\frac{\operatorname{Var}\left(R^{* *} \mid G\right)}{E\left(R^{* *} \mid G\right)-R_{0}} \in \mathbb{R}
$$

In this case, we can span the RE frontier with a safe asset by means of fixed-weight portfolios in the safe asset and the tangency portfolio.

2. The CE tangency portfolio is UE when

$$
\frac{E\left(R^{* 2} \mid G\right)-R_{0} E\left(R^{*} \mid G\right)}{E\left(R^{*} \mid G\right)-R_{0}\left(1-E\left(\mathfrak{A}^{+} \mid G\right)\right)} \in \mathbb{R}
$$

Nevertheless, we cannot represent the UE frontier with a safe asset by means of fixedweight portfolios in the safe asset and the tangency portfolio unless additionally $R_{0} \in \mathbb{R}$.

The results are easier to understand in the case of a single risky asset with return $R$ and excess return $A=R-R_{0}$. In that case, $R^{*}=R^{* *}=R$ and $\mathfrak{A}^{+}=\mathfrak{A}^{++}=0$, and hence the $\mathrm{CE}, \mathrm{UE}$, and RE frontiers without a safe asset are equal and given by a single point, $R$ itself. However, the CE, UE, and RE frontiers with a safe asset are different. $R$ will be also on the $\mathrm{CE}$ frontier with a safe asset, being the tangency portfolio, like in the Markowitz framework; and $A$ will be a CE excess return. However, $R$ is not necessarily on the UE or RE frontiers. Note the connection between these results and Corollary 3 . 
Panel A. Descritive statistics

$\begin{array}{lccccc} & \text { Mean } & \text { SD } & \text { Uncon. SR } & \text { Residual SD } & \text { Residual SR } \\ \text { MMR } & 7.3886 & 18.8474 & 0.392 & 17.4545 & 0.4233 \\ \text { SMB } & 3.2191 & 13.3611 & 0.2409 & 13.0297 & 0.2471 \\ \text { HML } & 5.5225 & 13.3059 & 0.415 & 12.9528 & 0.4264 \\ \text { WML } & 9.2816 & 17.7686 & 0.5224 & 14.4335 & 0.6431\end{array}$

Panel B. Predictive regressions

$\begin{array}{lcccccc} & \text { Constant } & \text { DP } & \text { DS } & \text { TS } & \mathrm{R}^{2} & \text { Wald } \\ \text { MMR } & 7.3886 & 6.5826 & 0.2186 & 4.2071 & 0.1423 & 0.0129 \\ \text { SMB } & 3.2191 & 1.5015 & 2.1041 & 0.1537 & 0.049 & 0.032 \\ \text { HML } & 5.5225 & 1.8824 & -0.8966 & 3.0908 & 0.0524 & 0.5772 \\ \text { WML } & 9.2816 & 2.5905 & -10.5476 & -1.0922 & 0.3402 & 0.0814 \\ & & & & & & \text { Joint }\end{array}$

Panel C. Weights of the representing portfolios

\begin{tabular}{lccccc} 
& \multicolumn{2}{c}{$\mathrm{A}^{+}$} & \multicolumn{2}{c}{ A++ } & Fixed \\
MMR & Mean & SD & Mean & SD & \\
& 0.0159 & 0.0138 & 0.0247 & 0.0235 & 0.0183 \\
MMR & & & & & 0.0162 \\
SMB & 0.0139 & 0.012 & 0.0298 & 0.0265 & 0.0074 \\
HML & 0.0062 & 0.0067 & 0.0115 & 0.0138 & 0.0258 \\
MMR & 0.0227 & 0.01 & 0.0428 & 0.0255 & 0.0158 \\
SMB & & & & & 0.0081 \\
HML & 0.0131 & 0.0083 & 0.0436 & 0.0288 & 0.0254 \\
WML & 0.0049 & 0.0052 & 0.0131 & 0.0128 & 0.0231
\end{tabular}

Table 1: Description of annual data.

Annual data from 1954 to 2010. MMR, SMB, HML and WML denote the Market, the Small-Minus-Big, the High-Minus-Low, and the Winners-Minus-Losers excess returns respectively. DP, DS, and TS denote the dividend price ratio, the default spread, and the term spread respectively. Panel A displays the mean, standard deviation, residual standard deviation, and the corresponding Sharpe ratios, of the four excess returns. Panel B shows the predictive regressions, where the three predictors have been standardized, with Wald tests of predictability (zero slopes). Panel C provides the mean and standard deviation of the representing portfolios weights for three subsets of returns, jointly with the fixed-weight counterpart of A+. Risk premia are linear in the predictors and conditional variances are constant. 
Uncon. SR

Uncon. Alpha

Zero-beta

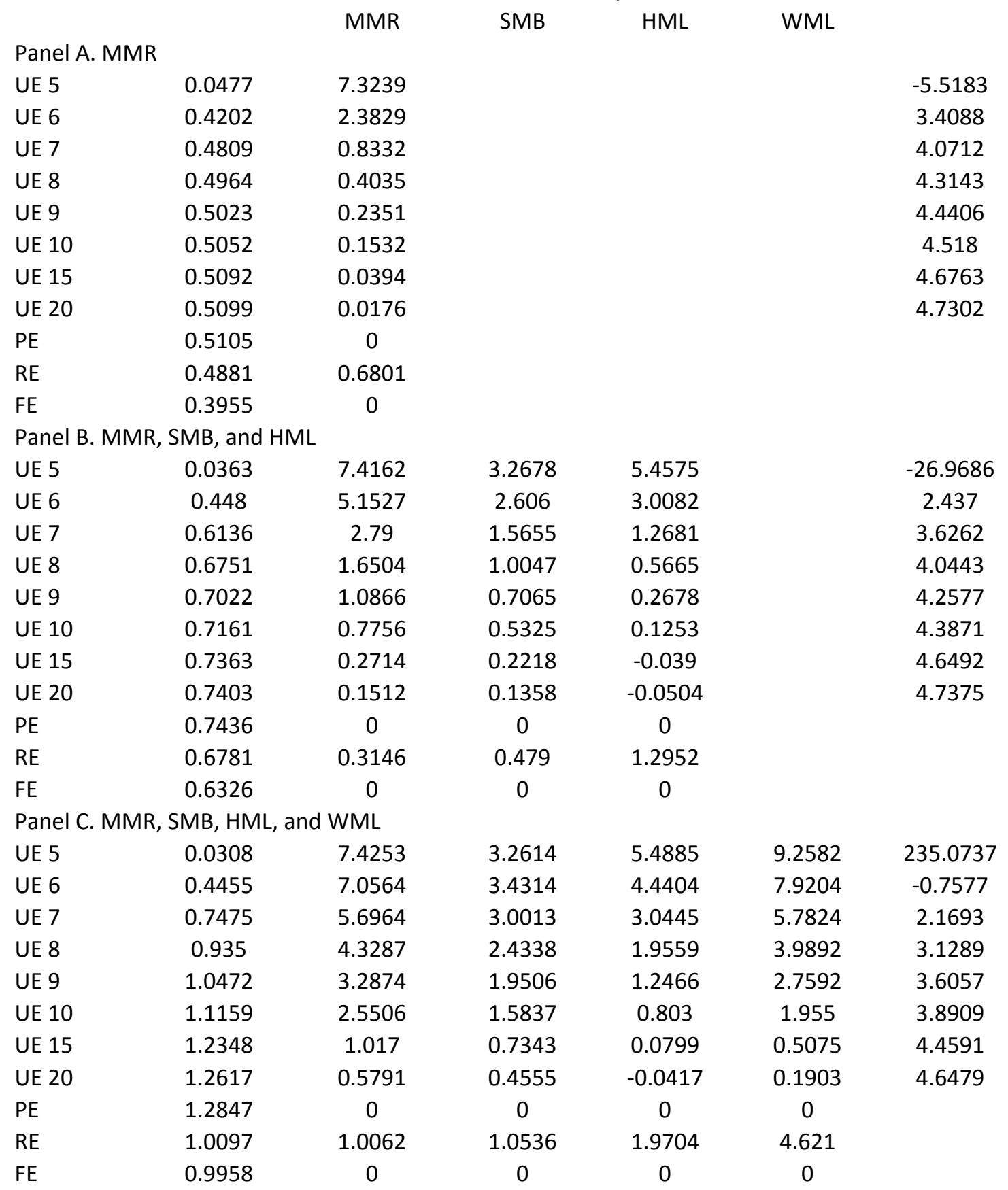

Table 2: Unconditional performance measures.

Annual data from 1954 to 2010. MMR, SMB, HML and WML denote the Market, the Small-Minus-Big, the High-Minus-Low, and the Winners-Minus-Losers excess returns respectively.. Panel A, B, and C represent three sets of available returns. We study unconditionally, performance, residually, and fixed-weight efficient returns, which we label as $\mathrm{UE}, \mathrm{PE}, \mathrm{RE}$, and $\mathrm{FE}$ respectively. We display several mean targets, from 5 to $20 \%$, for UE returns, and the last column shows their zero-beta returns. Unconditional alphas are computed for each one of the primitive excess returns. Risk premia are linear in the predictors and conditional variances are constant. 
Residual SR

\begin{tabular}{|c|c|c|c|c|c|}
\hline \multirow{2}{*}{\multicolumn{2}{|c|}{ Panel A. MMR }} & MMR & SMB & HML & WML \\
\hline & \multicolumn{5}{|c|}{ Panel A. MMR } \\
\hline UE 5 & 0.0604 & 7.26 & & & \\
\hline UE 6 & 0.4746 & 2.0388 & & & \\
\hline UE 7 & 0.5342 & 0.7218 & & & \\
\hline UE 8 & 0.5503 & 0.3538 & & & \\
\hline UE 9 & 0.557 & 0.2036 & & & \\
\hline UE 10 & 0.5604 & 0.1274 & & & \\
\hline UE 15 & 0.5659 & 0.0112 & & & \\
\hline UE 20 & 0.5673 & -0.0157 & & & \\
\hline PE & 0.5693 & -0.0502 & & & \\
\hline RE & 0.5902 & 0 & & & \\
\hline FE & 0.4271 & 0 & & & \\
\hline \multicolumn{6}{|c|}{ Panel B. MMR, SMB, and HML } \\
\hline UE 5 & 0.0517 & 7.4001 & 3.268 & 5.4278 & \\
\hline UE 6 & 0.5699 & 4.6171 & 2.2108 & 2.1934 & \\
\hline UE 7 & 0.7254 & 2.6709 & 1.2081 & 0.5704 & \\
\hline UE 8 & 0.7761 & 1.8992 & 0.7673 & 0.0316 & \\
\hline UE 9 & 0.7975 & 1.5433 & 0.5492 & -0.1812 & \\
\hline UE 10 & 0.8085 & 1.3518 & 0.4253 & -0.2796 & \\
\hline UE 15 & 0.8247 & 1.043 & 0.2062 & -0.3914 & \\
\hline UE 20 & 0.8282 & 0.9675 & 0.1447 & -0.3997 & \\
\hline$P E$ & 0.832 & 0.8649 & 0.0437 & -0.3685 & \\
\hline RE & 0.8895 & 0 & 0 & 0 & \\
\hline $\mathrm{FE}$ & 0.7011 & 0.6253 & -0.0908 & -0.3648 & \\
\hline \multicolumn{6}{|c|}{ Panel C. MMR, SMB, HML, and WML } \\
\hline UE 5 & 0.0532 & 7.4415 & 3.2821 & 5.4731 & 9.2455 \\
\hline UE 6 & 0.7204 & 6.3963 & 3.1123 & 3.5144 & 6.3864 \\
\hline UE 7 & 1.0632 & 4.4456 & 2.1351 & 1.6632 & 3.2468 \\
\hline UE 8 & 1.2084 & 3.2359 & 1.4359 & 0.7695 & 1.6093 \\
\hline UE 9 & 1.2744 & 2.5705 & 1.0189 & 0.3666 & 0.8166 \\
\hline UE 10 & 1.3081 & 2.1859 & 0.7629 & 0.1743 & 0.4079 \\
\hline UE 15 & 1.3537 & 1.542 & 0.2894 & -0.0245 & -0.1266 \\
\hline UE 20 & 1.3608 & 1.3871 & 0.1569 & -0.0214 & -0.1932 \\
\hline PE & 1.3611 & 1.1965 & -0.0493 & 0.1001 & -0.1318 \\
\hline RE & 1.4901 & 0 & 0 & 0 & 0 \\
\hline FE & 1.1607 & -0.3591 & -1.5127 & -0.8112 & 1.6671 \\
\hline
\end{tabular}

\begin{tabular}{lll}
\multicolumn{2}{c}{ Residual Alpha } & \\
$\mathrm{SMB}$ & $\mathrm{HML}$ & $\mathrm{WML}$
\end{tabular}

Table 3: Residual performance measures. 
Panel A. Descritive statistics

$\begin{array}{lccccc} & \text { Mean } & \text { SD } & \text { Uncon. SR } & \text { Residual SD } & \text { Residual SR } \\ \text { MMR } & 6.5884 & 15.2762 & 0.4313 & 15.1166 & 0.4358 \\ \text { SMB } & 2.6207 & 10.3458 & 0.2533 & 10.2844 & 0.2548 \\ \text { HML } & 4.7411 & 9.6571 & 0.4909 & 9.6409 & 0.4918 \\ \text { WML } & 8.9353 & 14.1838 & 0.63 & 14.0644 & 0.6353\end{array}$

Panel B. Predictive regressions

$\begin{array}{lcccccc} & \text { Constant } & \text { DP } & \text { DS } & \text { TS } & R^{2} & \text { Wald } \\ \text { MMR } & 0.549 & 0.5523 & -0.1438 & 0.5663 & 0.0208 & 0.0013 \\ \text { SMB } & 0.2184 & 0.1277 & 0.1732 & 0.2035 & 0.0118 & 0.0372 \\ \text { HML } & 0.3951 & 0.0654 & -0.1753 & 0.0957 & 0.0033 & 0.7833 \\ \text { WML } & 0.7446 & 0.1043 & -0.5338 & -0.0737 & 0.0168 & 0.4431 \\ & & & & & & \text { Joint } \\ & & & & & & 0.0021\end{array}$

Panel C. Weights of the representing portfolios

\begin{tabular}{lccccc} 
& \multicolumn{2}{c}{ A+ } & A++ & Fixed \\
MMR & Mean & SD & Mean & SD & \\
SMB & 0.037 & 0.0373 & 0.0443 & 0.0468 & 0.0345 \\
HML & 0.0267 & 0.0407 & 0.032 & 0.0502 & 0.0233 \\
& 0.088 & 0.0489 & 0.1004 & 0.0593 & 0.0689 \\
MMR & & & & & 0.0419 \\
SMB & 0.034 & 0.0357 & 0.0462 & 0.0496 & 0.0242 \\
HML & 0.0333 & 0.0448 & 0.0427 & 0.0601 & 0.0841 \\
WML & 0.0993 & 0.052 & 0.131 & 0.0777 & 0.0559
\end{tabular}

Table A1: Description of monthly data.

Monthly data from 1954 to 2010. MMR, SMB, HML and WML denote the Market, the SmallMinus-Big, the High-Minus-Low, and the Winners-Minus-Losers excess returns respectively. DP, DS, and TS denote the dividend price ratio, the default spread, and the term spread respectively. Panel A displays the annualized mean, standard deviation, residual standard deviation, and the corresponding Sharpe ratios, of the four excess returns. Panel B shows the predictive regressions, where the three predictors have been standardized, with Wald tests of predictability (zero slopes). Panel C provides the mean and standard deviation of the representing portfolios weights for two subsets of returns, jointly with the fixed-weight counterpart of A+. Risk premia are linear in the predictors and conditional variances follow a DCC model. 
Uncon. SR

Uncon. Alpha

SMB HML
Zero-beta

WML

4.1321

4.4441

4.5602

4.6209

4.6581

4.7347

4.7608

$\mathrm{PE}$

1.1396

$-0.0094$

0.0108

$-0.0153$

$-0.0115$

$-0.0075$

0.0068

$-0.0086$

$$
0
$$

0

$-0.0201$

0.0054

0.3431

FE

1.133

0.163

0.0648

0.1173

Panel B. MMR, SMB, HML, and WML

$\begin{array}{lcccccc}\text { UE 5 } & 0.6107 & 5.6443 & 2.4457 & 3.9955 & 7.8623 & -8.4612 \\ \text { UE 6 } & 1.5501 & 1.0242 & 0.6336 & 0.6624 & 1.6231 & 3.3762 \\ \text { UE 7 } & 1.6528 & 0.3187 & 0.2677 & 0.1828 & 0.578 & 4.0803 \\ \text { UE 8 } & 1.679 & 0.1408 & 0.1562 & 0.0683 & 0.2946 & 4.334 \\ \text { UE 9 } & 1.6892 & 0.074 & 0.1067 & 0.0277 & 0.1803 & 4.4648 \\ \text { UE 10 } & 1.6942 & 0.0427 & 0.0798 & 0.01 & 0.123 & 4.5445 \\ \text { UE 15 } & 1.7012 & 0.0035 & 0.0337 & -0.0081 & 0.0382 & 4.7069 \\ \text { UE 20 } & 1.7025 & -0.0019 & 0.021 & -0.0085 & 0.0199 & 4.762 \\ \text { PE } & 1.7037 & 0 & 0 & 0 & 0 & \\ \text { RE } & 1.6853 & 0.1198 & 0.2578 & 0.322 & 0.5388 & \\ \text { FE } & 1.1209 & 0.5909 & 0.235 & 0.4252 & 0.8013 & \end{array}$

Table A2: Unconditional performance measures.

Monthly data from 1954 to 2010, the numbers in the table are annualized. MMR, SMB, HML and WML denote the Market, the Small-Minus-Big, the High-Minus-Low, and the Winners-Minus-Losers excess returns respectively. Panel A and B represent two sets of available returns. We study unconditionally, performance, residually, and fixed-weight efficient returns, which we label as $U E, P E, R E$, and FE respectively. We display several mean targets, from 5 to 20\%, for UE returns, and the last column shows their zero-beta returns. Unconditional alphas are computed for each one of the primitive excess returns. Risk premia are linear in the predictors and conditional variances follow a DCC model. 
Residual SR

\begin{tabular}{llrl}
\multicolumn{4}{c}{ Residual Alpha } \\
MMR & SMB & HML
\end{tabular}

Panel A. MMR, SMB, and HML

$\begin{array}{lcccc}\text { UE 5 } & 0.7158 & 3.8983 & 1.779 & 2.6463 \\ \text { UE 6 } & 1.1468 & 0.3038 & 0.2124 & -0.1185 \\ \text { UE 7 } & 1.1677 & 0.135 & 0.0994 & -0.2453 \\ \text { UE 8 } & 1.1726 & 0.1037 & 0.0687 & -0.268 \\ \text { UE 9 } & 1.1745 & 0.0949 & 0.0554 & -0.2741 \\ \text { UE 10 } & 1.1754 & 0.0921 & 0.0482 & -0.2757 \\ \text { UE 15 } & 1.1768 & 0.0929 & 0.036 & -0.2742 \\ \text { UE 20 } & 1.1771 & 0.0951 & 0.0325 & -0.2721 \\ \text { PE } & 1.1774 & 0.1029 & 0.0268 & -0.2654 \\ \text { RE } & 1.1836 & 0 & 0 & 0 \\ \text { FE } & 0.8035 & 0.3191 & -0.225 & -0.0836\end{array}$

Panel B. MMR, SMB, HML, and WML

$\begin{array}{lccccc}\text { UE 5 } & 0.6963 & 5.4902 & 2.3554 & 3.8696 & 7.73 \\ \text { UE 6 } & 1.6526 & 0.7488 & 0.3317 & 0.3118 & 1.0641 \\ \text { UE 7 } & 1.7354 & 0.1855 & 0.0125 & -0.0925 & 0.1409 \\ \text { UE 8 } & 1.7541 & 0.0613 & -0.0762 & -0.1774 & -0.0932 \\ \text { UE 9 } & 1.7606 & 0.0202 & -0.1134 & -0.2036 & -0.1837 \\ \text { UE 10 } & 1.7634 & 0.0037 & -0.1329 & -0.2132 & -0.2276 \\ \text { UE 15 } & 1.7664 & -0.009 & -0.1641 & -0.2167 & -0.2884 \\ \text { UE 20 } & 1.7665 & -0.0066 & -0.172 & -0.2128 & -0.2998 \\ \text { PE } & 1.7653 & 0.0091 & -0.1835 & -0.1968 & -0.3082 \\ \text { RE } & 1.7804 & 0 & 0 & 0 & 0 \\ \text { FE } & 1.1329 & -0.2926 & -0.1487 & 0.1856 & 0.0046\end{array}$

Table A3: Residual performance measures.

Monthly data from 1954 to 2010, the numbers in the table are annualized. MMR, SMB, HML and WML denote the Market, the Small-Minus-Big, the High-Minus-Low, and the WinnersMinus-Losers excess returns respectively. Panel A and B represent two sets of available returns. We study unconditionally, performance, residually, and fixed-weight efficient returns, which we label as UE, PE, RE, and FE respectively. We display several mean targets, from 5 to $20 \%$, for UE returns. Residual alphas are computed for each one of the primitive excess returns. Risk premia are linear in the predictors and conditional variances are constant. 
Figure 1: Weights of the representing portfolios.

The first column shows the weights of the uncentred representing portfolio $A+$, and the weights of $A++$ can be found in the second column. The first row displays the weights when only MMR is available, the second row adds SMB and HML, and the third row also includes WML. The weights on MMR, SMB, HML, and WML are blue, green, red, and cyan respectively.
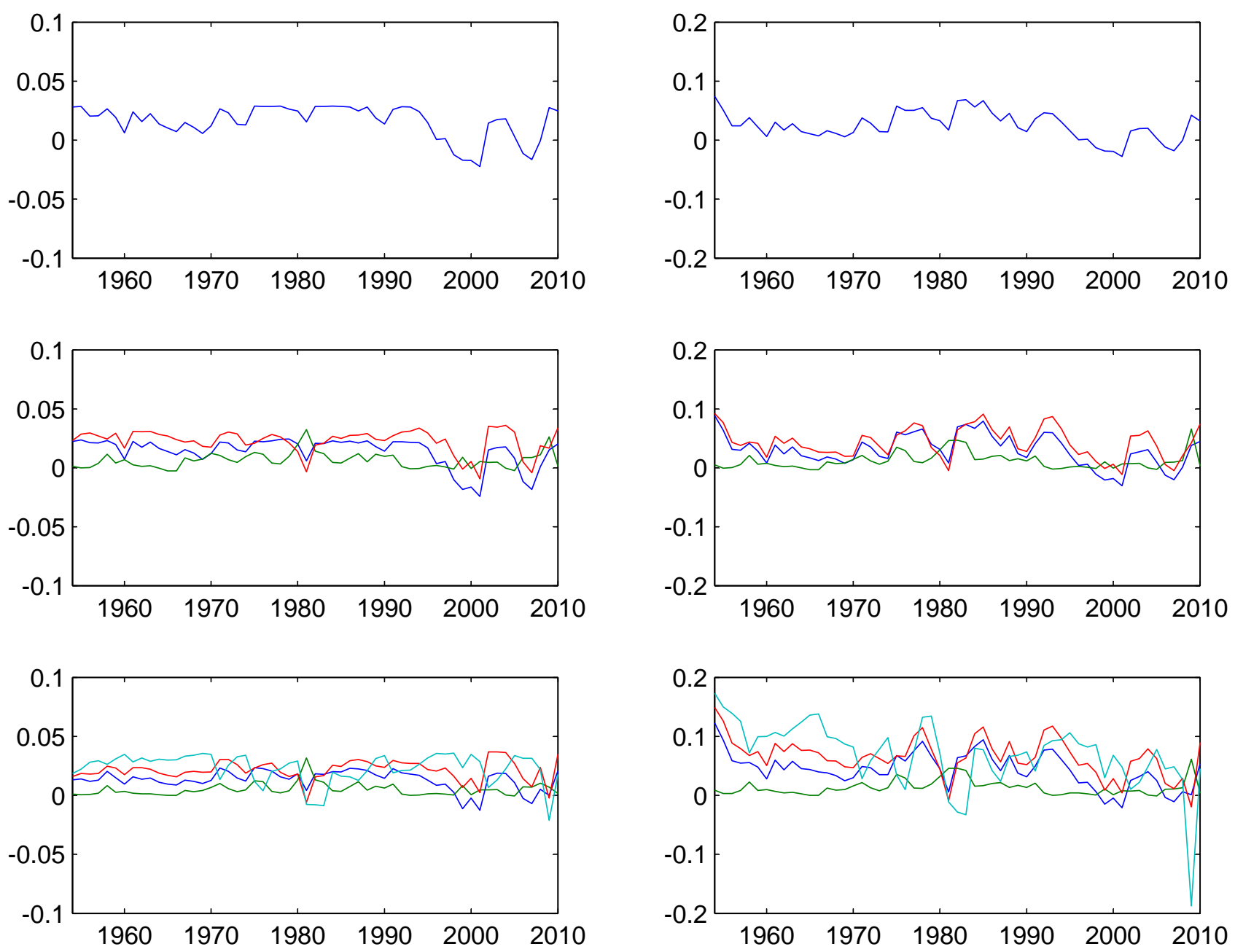
Figure 2: Safe asset return and conditional Sharpe ratio of CE returns.

The safe asset return is shown as an annual \% in the first plot. The second plot shows he conditional Sharpe ratio for three sets of excess returns: MMR (blue), adding SMB and HML (green), and also adding WML (red).
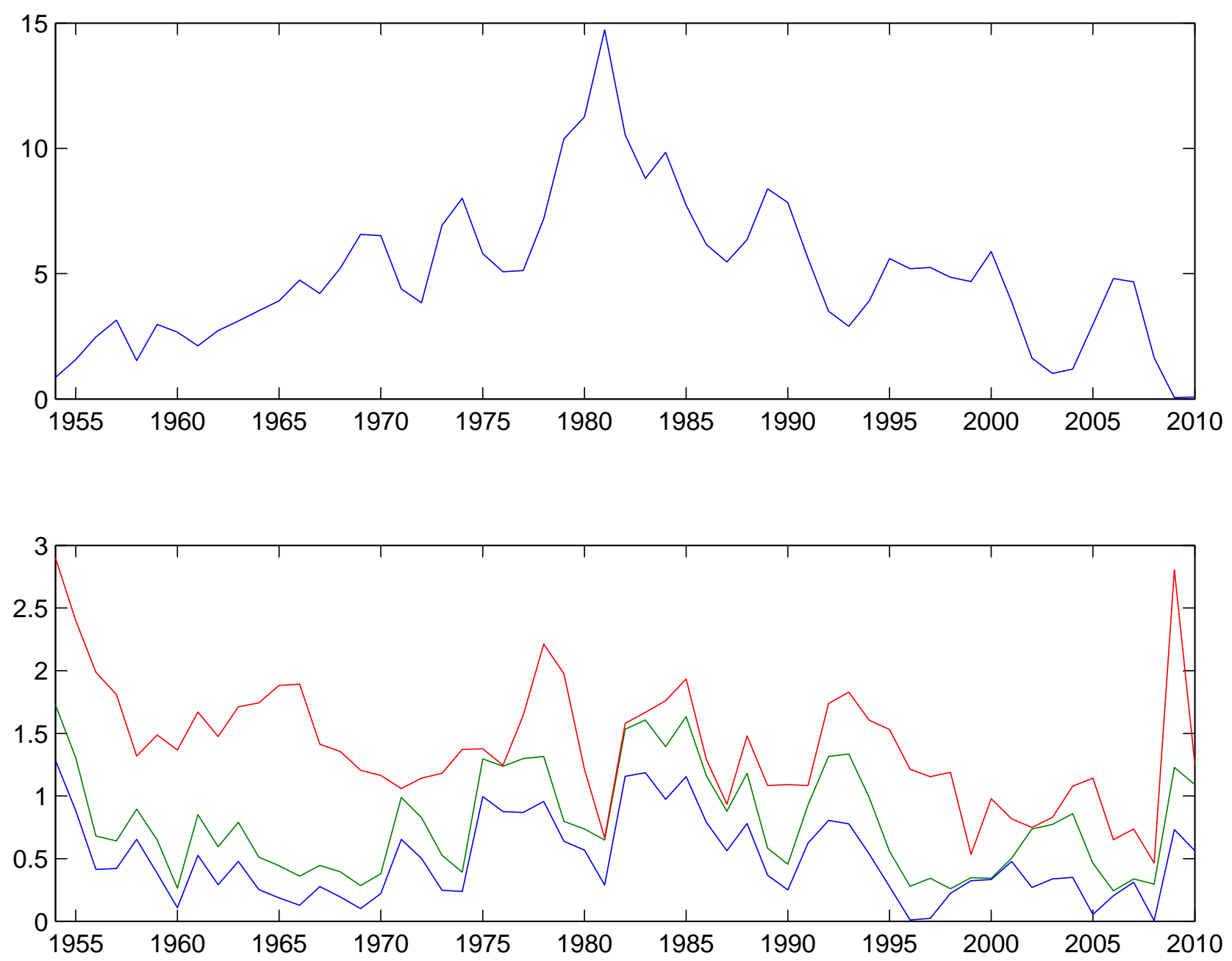
Figure 3: UE and PE returns.

The first column shows the mean-variance frontiers in space $\left[(\operatorname{Var}(A))^{1 / 2}, E(A)\right]$, and their representation in space $\left[(\operatorname{Var}(R))^{1 / 2}, E(R)\right]$ can be found in the second column. Both means and standard deviations are measured in annual \%. The first row displays the frontiers when only MMR is available, the second row adds SMB and HML, and the third row also includes WML. UE and PE returns are blue and green respectively. For comparison, efficient fixed-weight returns are also shown in red.
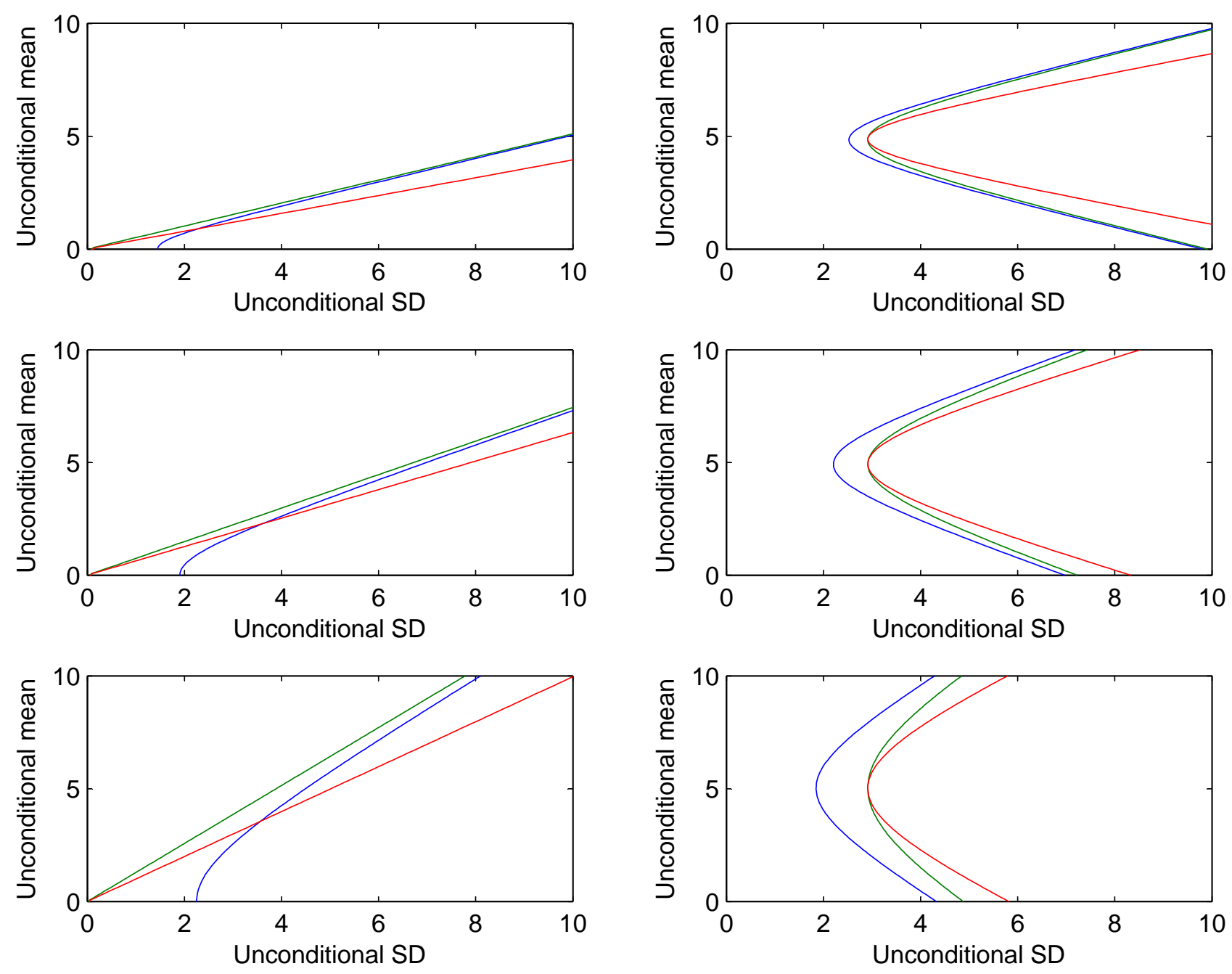
Figure 4: UE, PE and RE returns.

The first column shows the mean-variance frontiers in space $\left[(E[\operatorname{Var}(R \mid G)])^{1 / 2}, E(R)\right]$, and their representation in space $\left[(\operatorname{Var}(\mathrm{R}))^{1 / 2}, E(R)\right]$ can be found in the second column. Both means and standard deviations are measured in annual \%. The first row displays the frontiers when only MMR is available, the second row adds SMB and HML, and the third row also includes WML. UE, PE, and RE returns are blue, green, and cyan respectively. For comparison, efficient fixed-weight returns are also shown in red.
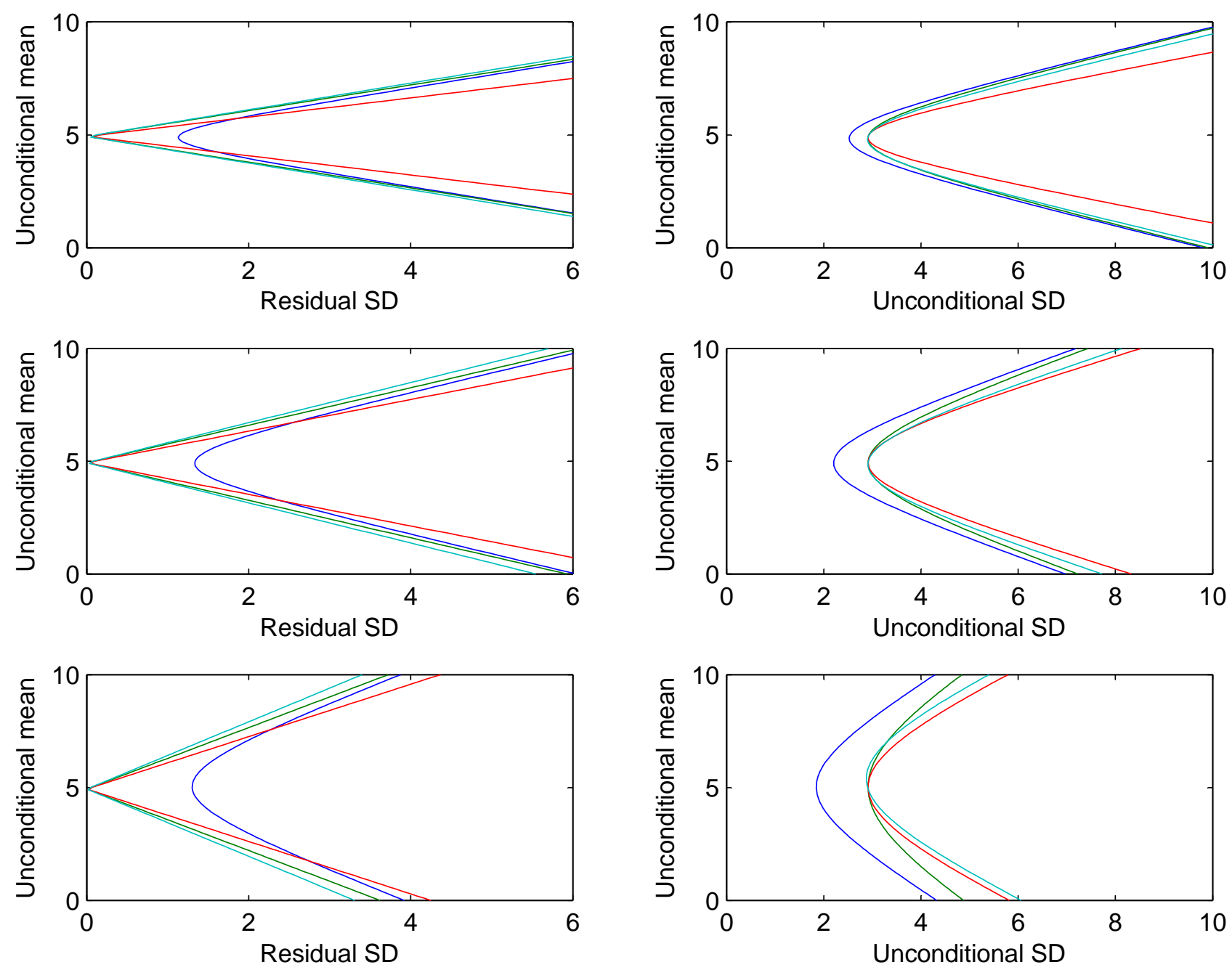
Figure 5: Scaling for efficient returns.

The first plot shows the scaling of $A+$ for different types of efficiency when the target return is $5 \%$, while the second plot displays the scaling for a target of $10 \%$. In this figure, $A+$ is constructed with MMR, SMB, and HML. UE, PE, and RE returns are blue, green, and cyan respectively.
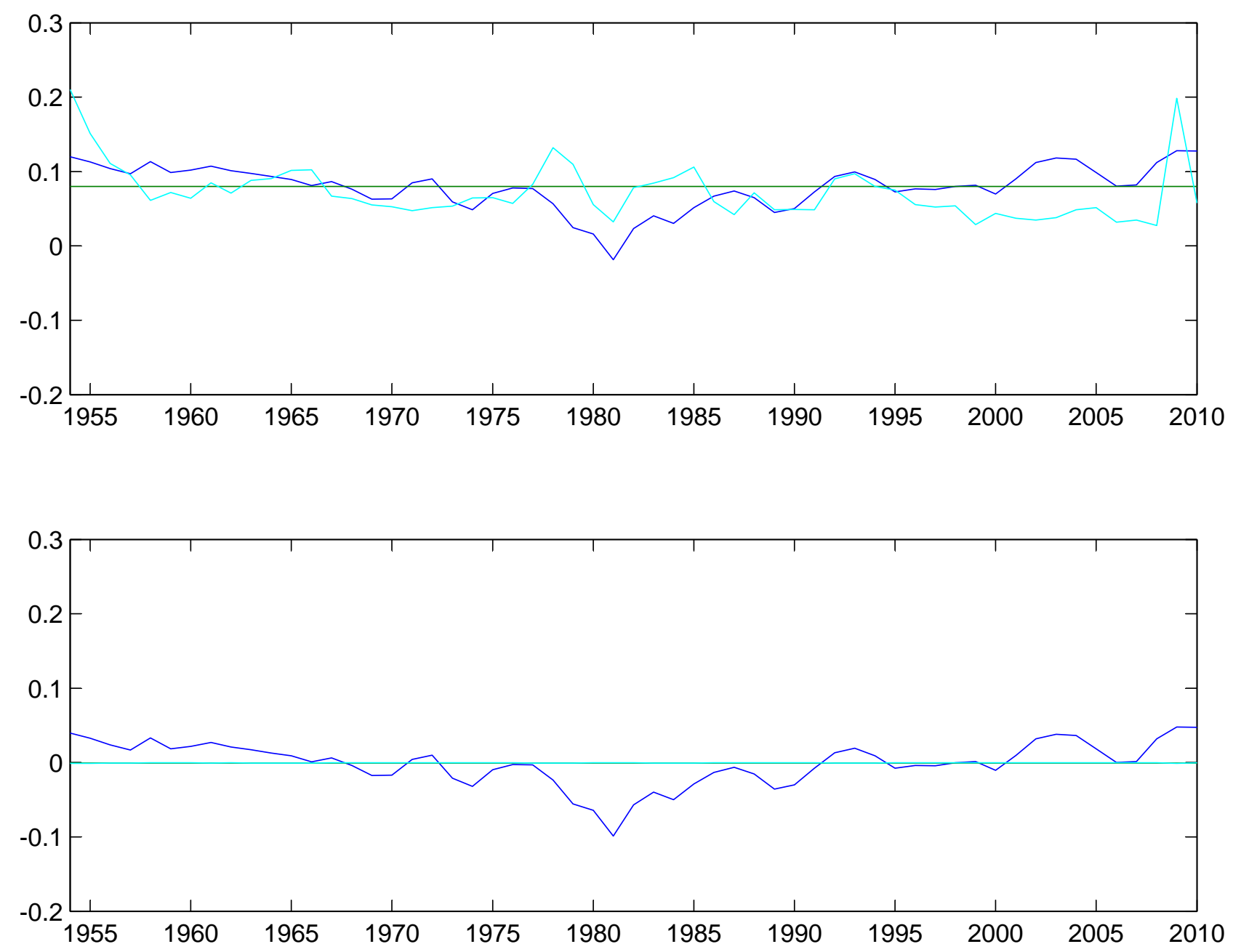
Figure 6: Summary of efficient excess and gross returns.

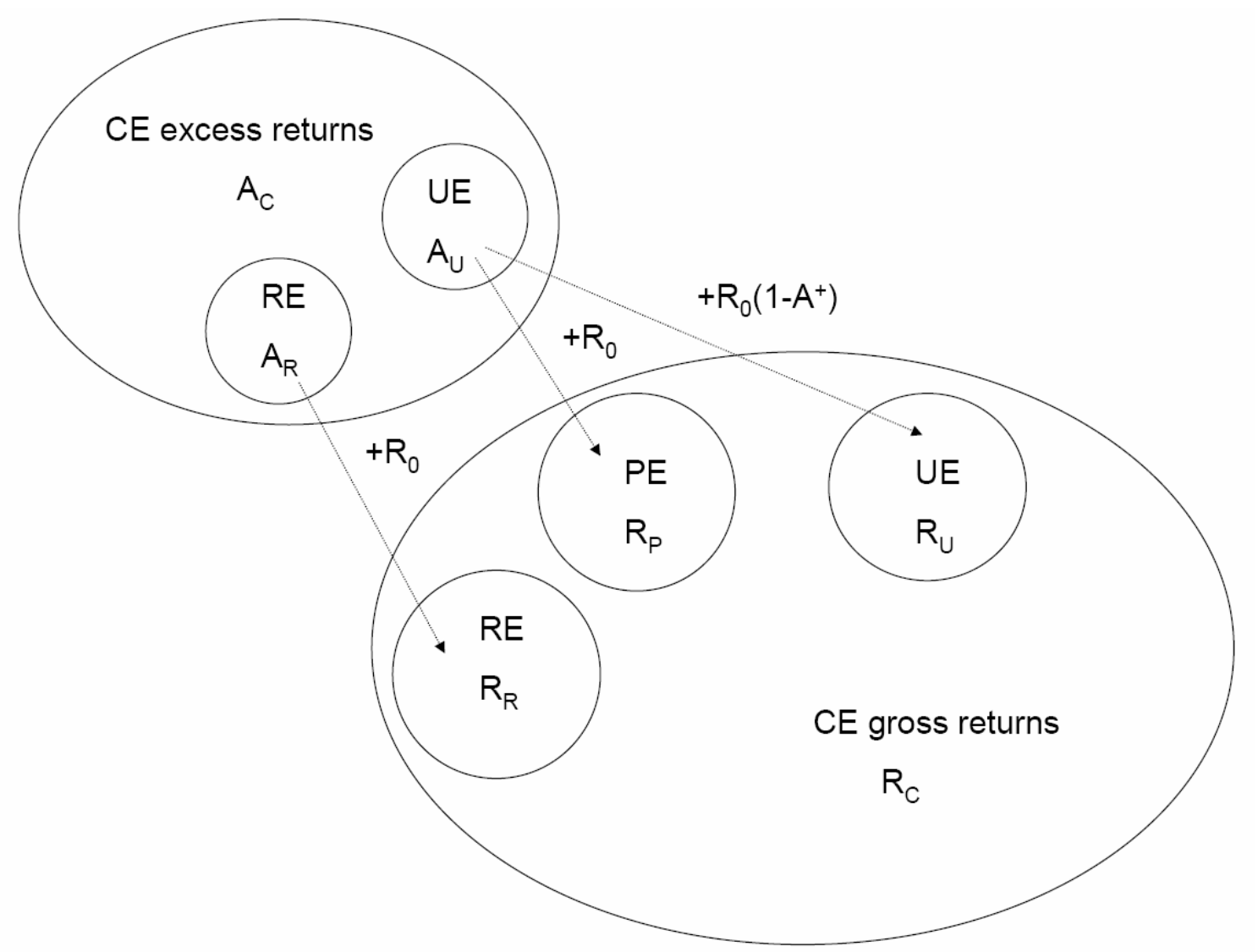

